$\mathrm{AJ}$, in press

\title{
The Molonglo Southern 4 Jy Sample (MS4). I. Definition
}

\author{
A. M. Burgess ${ }^{1}$ \\ annb@psych.usyd.edu.au \\ and \\ R. W. Hunstead \\ School of Physics, University of Sydney, NSW 2006, Australia \\ rwh@physics .usyd.edu.au
}

\begin{abstract}
We have defined a complete sample of 228 southern radio sources at $408 \mathrm{MHz}$ with integrated flux densities $S_{408}>4.0 \mathrm{Jy}$, Galactic latitude $|b|>10^{\circ}$ and declination $-85^{\circ}<\delta<-30^{\circ}$. The main finding survey used was the Molonglo Reference Catalogue. We describe in detail how the Molonglo Southern 4 Jy sample (MS4) was assembled and its completeness assessed. Sources in the sample were imaged at $843 \mathrm{MHz}$ with the Molonglo Observatory Synthesis Telescope to obtain positions accurate to about $1^{\prime \prime}$, as well as flux densities and angular sizes; follow-up radio and optical observations are presented in Paper II. Radio spectra for the MS4 have been compiled from the literature and used to estimate flux densities at $178 \mathrm{MHz}$. The strong-source subset of MS4, with $S_{178}>10.9 \mathrm{Jy}$ (SMS4), provides a southern sample closely equivalent to the well-studied northern 3CRR sample. Comparison of SMS4 with 3CRR shows a reassuring similarity in source density and median flux density between the two samples.
\end{abstract}

Subject headings: radio continuum: galaxies — galaxies: active — surveys 


\section{INTRODUCTION}

Well defined, complete surveys are essential starting points for learning about the global characteristics of astronomical sources. At radio frequencies, the easiest way to select a large, well defined sample is to choose all objects brighter than a given flux density at a given observing frequency. Such a sample has the advantage of containing the objects with the highest signal-to-noise ratio. Because any one finding survey is likely to be affected either by confusion or by over-resolution for some of the sources, it is often necessary to use more than one survey to define a flux density-limited sample. Even then the sample may not be complete, but may, for example, be missing sources (or portions of sources) of low surface brightness or very large angular size.

One of the best studied radio samples in the past few decades has been the northern hemisphere Third Cambridge Catalogue (3C; Edge et al. 1959) and its revised versions the 3CR (Bennett 1962) and 3CRR (Laing et al. 1983). Being selected at low frequency (159 and later $178 \mathrm{MHz}$ ), it contains a large proportion of extended, steep-spectrum sources. Because of the high flux-density limit $\left(S_{178}\right)$ of around $11 \mathrm{Jy}$, the sample contains a large proportion of very powerful sources. The most complete version, the $3 \mathrm{CRR}$, is the one discussed in this paper.

As so many studies of high-power radio sources have been based on this one sample, it is essential to have a comparison sample to test whether the 3CRR is truly representative. This paper is concerned with the definition of such a comparison sample, selected at low frequency to have properties similar to 3CRR. Increasing the sky coverage is more important at high flux densities where the total number of sources is limited. This sample was selected at $408 \mathrm{MHz}$ to contain the $\sim 200$ brightest extragalactic objects south of $\delta=-30^{\circ}$.

The sample is defined in Section 2. Follow-up observations of the whole sample with the Molonglo Observatory Synthesis Telescope at $843 \mathrm{MHz}$ are described in Section 3. These observations have provided more accurate positions and angular sizes, as well as flux densities

in a gap in the radio spectrum. The definition of a strong subsample at $178 \mathrm{MHz}$, for comparison with 3CRR, is described in Section 4. In Paper II (Burgess \& Hunstead 2006) we present follow-up imaging at $5 \mathrm{GHz}$ of the more compact sources with the Australia Telescope Compact Array, together with optical identifications using the UK Schmidt sky survey and CCD images from the Anglo-Australian Telescope. 


\section{DEFINITION OF THE MS4 SAMPLE}

Our motivation was to generate a sample of strong southern radio sources for comparison with (and extension of) the well studied northern 3CRR sample. For reference, Table 1 contains a summary of southern finding surveys of strong sources. Our selection frequency of $408 \mathrm{MHz}$ (as opposed to $178 \mathrm{MHz}$ used for 3CRR) was chosen because it allowed the sources to be selected from the Molonglo Reference Catalogue (MRC; Large et al. 1981), the deepest and most complete low-frequency survey of the southern sky. The flux-density limit of $4.0 \mathrm{Jy}$ was chosen to give a useful but manageable number of sources, $\sim 200-250$. The sample is therefore called the Molonglo Southern 4 Jy sample, abbreviated as MS4.

The selection criteria for the MS4 sample are as follows:

(i) Declination $-85^{\circ}<\delta<-30^{\circ}$.

(ii) Flux density $S_{408}>4.0 \mathrm{Jy}$.

(iii) Galactic latitude $|b|>10^{\circ}$.

(iv) Not in the Magellanic Cloud regions.

(v) Not a known Galactic source.

The reason for excluding the small area south of $-85^{\circ}$ is that the MRC is incomplete in this region. At least one source would otherwise be in the sample: PKS B0349-88, with $S_{408}=6.8 \mathrm{Jy}$ (Price \& Milne 1965). The northern limit of $\delta=-30^{\circ}$ was chosen to preserve a reasonable beamshape for follow-up images made with the east-west arrays of the Australia Telescope Compact Array (ATCA) and Molonglo Observatory Synthesis Telescope (MOST). For the purposes of sample definition the original B1950 coordinates were retained, but all positions are given for the J2000 equinox.

The Galactic Plane and Magellanic Cloud regions are excluded for two reasons: the strong radio emission from local sources makes background extragalactic objects hard to recognise, and the dust obscuration and high star density make optical identifications difficult. The Magellanic Cloud regions are those defined by Turtle \& Amy (1991), i.e., $00^{\mathrm{h}} 25^{\mathrm{m}}<\alpha<01^{\mathrm{h}} 35^{\mathrm{m}},-74^{\circ} 40^{\prime}<\delta<-70^{\circ} 45^{\prime}$ for the Small Magellanic Cloud, and

$04^{\mathrm{h}} 46^{\mathrm{m}}<\alpha<05^{\mathrm{h}} 58^{\mathrm{m}},-72^{\circ} 20^{\prime}<\delta<-65^{\circ} 10^{\prime}$ for the Large Magellanic Cloud. Examination of the $408 \mathrm{MHz}$ MC4 survey (Clarke et al. 1976) reveals that the only sources in these regions with $S_{408}>4.0 \mathrm{Jy}$ are associated with the Clouds rather than being background objects. From the mean source density of MS4 (§2.6) we would expect only two sources to lie in these regions, so a count of zero is not surprising. 


\subsection{The Molonglo Cross Telescope and the Molonglo Reference Catalogue}

The Molonglo Reference Catalogue (MRC) was compiled from data taken between 1968 and 1978 with the Molonglo Cross Telescope. This telescope was a Mills Cross interferometer operating at $408 \mathrm{MHz}$ with a bandwidth of $2.5 \mathrm{MHz}$, and resolution of $2.67^{\prime} \times 2.86^{\prime} \sec (\delta+$ $35^{\circ} .4$ ) (Mills et al. 1963). The flux-density calibration (Hunstead 1991) was based on the absolute scale of Wyllie (1969a) and the pencil-beam sensitivity curve measured by Hunstead (1972). The survey for the MRC covered the declination range $-85^{\circ} \leq \delta \leq+18^{\circ} .5$, with Galactic latitude $|b|>3^{\circ}$. The MRC contains 12141 sources above its lower flux-density limit of $S_{408}=0.7 \mathrm{Jy}$, and contains 7347 sources above its notional completeness level of 1 Jy. As well as positions and flux densities, the catalogue contains cross-references to other catalogues, and some structural information for extended sources. For sources stronger than 4 Jy the positional errors are around $3^{\prime \prime}$ in $\alpha$ and $4^{\prime \prime}$ in $\delta$, and the standard error in flux density is around $4 \%$.

In defining our sample, we used the MRC in preference to the two previous extensive low-frequency surveys of the southern sky, the Mills, Slee, and Hill (MSH) survey at 85.5 MHz (Mills et al. 1958, 1960, 1961) and the Parkes $408 \mathrm{MHz}$ catalogues (Bolton et al. 1964; Price \& Milne 1965). Compared with the MRC, the MSH and Parkes catalogues have less uniform sky coverage, lower sensitivity, and higher source confusion due to lower resolution. Of the 228 sources in the MS4 sample, 43 were not in the original Parkes survey: of these, 35 were in the region of sky covered by that survey. Because of their much wider beams, the Parkes and MSH surveys were valuable in providing integrated flux densities for strong but very extended sources (such as the lobes of Centaurus A) which are substantially resolved out by the Molonglo Cross.

\subsection{Initial Selection of the Sample}

Initial selection of the MS4 sample from the MRC gave 215 sources in the region of

interest with $S_{408}>4.0 \mathrm{Jy}$. This list was refined using the study of Jones \& McAdam (1992), hereafter referred to as JM92. They used the Molonglo Observatory Synthesis Telescope (MOST) to image at $843 \mathrm{MHz}$ all MRC sources south of $-30^{\circ}$ which were flagged as extended or multiple. Their higher resolution MOST images made it possible to distinguish between genuine extended sources, some with more than one MRC entry, and close groups of unrelated sources. Following Jones (1989b), two MRC sources were considered to be parts of a single source if they were extended towards each other, or if they were connected at or above the $2 \%$ contour level. This latter criterion corresponds to a separation of about $105^{\prime \prime}$ for two unresolved sources. For instance, we excluded MRC B0230-666, with $S_{408}=4.39$ Jy, from 
our sample because JM92 show it is composed of two separate compact sources, each well below our 4 Jy limit. MRC B1459-417, identified with the Galactic supernova remnant SNR 1006 (Stephenson et al. 1977), was also excluded. After excluding these two sources, and counting 5 sources with multiple MRC entries only once, our sample contained 208 sources. This was, however, still incomplete, because of the underestimation of flux densities of extended sources in the MRC.

The MRC flux densities were calculated using a point-source fitting algorithm which is reliable for sources with angular extent less than $\sim 1^{\prime}$. For larger angular sizes the fitted value underestimates the integrated flux density, so it was necessary to estimate the integrated $S_{408}$ for each extended source in the region of interest. We first obtained a candidate list of 178 sources with MRC flux densities between 1 and $4 \mathrm{Jy}$, and flagged as extended or multiple; weaker sources were ignored as the $\mathrm{MRC}$ is incomplete below $1 \mathrm{Jy}$. Of the 178 sources, 97 were found by JM92 to have largest angular size $>1^{\prime}$. We searched the literature for flux-density measurements at $408 \mathrm{MHz}$ for these 97 sources. If such measurements were unavailable, we used the radio spectrum or unpublished Molonglo $408 \mathrm{MHz}$ data. As a result of these estimates, described in the following section, 20 extended sources were added to the sample, bringing the total number of sources to 228.

\subsection{Flux Densities of Extended Sources}

\subsubsection{Published Flux Densities at $408 \mathrm{MHz}$}

$408 \mathrm{MHz}$ flux densities for extended southern sources are available from either the $64 \mathrm{~m}$ Parkes Telescope or the Molonglo Cross. The Molonglo papers used the absolute fluxdensity scale of Wyllie (1969a), whereas the Parkes catalogues used the scale of Conway et al. (1963), which was nominally about 10\% lower. However, Wyllie (1969b) found a mean ratio $\langle$ Molonglo/Parkes $=1.000 \pm 0.015$ and we have therefore not made any adjustments to the Parkes flux densities.

The main Parkes $408 \mathrm{MHz}$ data were collected as part of the original Parkes surveys (Bolton et al. 1964; Price \& Milne 1965). The Parkes beam size of $48^{\prime}$ often led to overestimation of flux densities because of confusing sources in the beam. We therefore used these flux densities only if estimates via the radio spectrum $(\S 2.3 .2)$ were unavailable, or

for strong sources where confusion was unlikely to be severe. Parkes $408 \mathrm{MHz}$ flux densities were used to exclude four sources from the sample. Few of the remaining candidates were in the Parkes catalogue, as they lay well below its stated completeness limit of $4 \mathrm{Jy}$.

The two main sets of Molonglo Cross data in the region of interest were those of Schilizzi 
\& McAdam (1975), hereafter referred to as SM75, and the MC4 survey of the Magellanic Cloud regions (Clarke et al. 1976). SM75 estimated integrated $408 \mathrm{MHz}$ flux densities for 116 sources found to be extended with Parkes or Molonglo. A total of 17 candidates from our list were in their survey, of which 11 were listed as having $S_{408}>4.0 \mathrm{Jy}$. One of these candidates, MRC B2130-538, in the cluster Abell3785, was subsequently excluded because a high-resolution image (Haigh 2000), made with the Australia Telescope Compact Array at $20 \mathrm{~cm}$, showed it to be two independent radio sources. We also included a source, PKS B1400-33, which had $S_{408}>4.0 \mathrm{Jy}$ in SM75, but did not appear in the MRC.

The MC4 finding survey covered the Magellanic Clouds, as well as comparison regions in the declination range $-61^{\circ} \leq \delta \leq-75^{\circ}$. Of the 10 candidates observed in this survey, two were found to have integrated flux density $S_{408}>4.0 \mathrm{Jy}$.

Integrated flux densities at $408 \mathrm{MHz}$ measured with the Molonglo Cross by J. Rathmell (Ekers et al. 1989) were used to include MRC B1056-360, with $S_{408}=4.09 \mathrm{Jy}$.

\subsubsection{Estimates from the Radio Spectrum}

Because extended sources usually have power-law radio spectra, it is often possible to estimate flux densities by linear interpolation or extrapolation of a $\log S_{\nu}$ : $\log \nu$ plot. We used flux-density measurements at other frequencies to estimate $S_{408}$ for sources without published integrated flux densities at $408 \mathrm{MHz}$. It was sometimes necessary to extrapolate to $S_{408}$, if data at lower frequencies were unavailable. There will be systematic error in these estimates both from missing flux density for sources with large angular extent, and from confusion in low-resolution observations, in particular those of $\mathrm{MSH}$ at $85.5 \mathrm{MHz}$, and Parkes at 408 and $1410 \mathrm{MHz}$.

To quantify the error in the flux-density estimates, we compared flux densities from SM75 with values interpolated or extrapolated from the radio spectrum for 20 extended sources. For 15 of these sources, the estimate from the radio spectrum was larger than the SM75 value. For the 8 sources with largest angular size LAS $<7^{\prime}$, the median flux-density difference was $13 \%$, and for the 12 sources with LAS $\geq 7^{\prime}$ the median flux-density difference was $24 \%$. These systematic differences are attributed to the limited surface brightness sensitivity of the $3^{\prime}$ Molonglo Cross pencil beam.

On the basis of the radio spectra, two sources were added to the sample. 


\subsubsection{Estimates from Unpublished Molonglo $408 \mathrm{MHz}$ Data}

The unpublished Molonglo Transit Catalogue, from an intermediate stage in the compilation of the MRC, contains estimates of integrated flux densities which we were able to compare with the corresponding values in SM75. The Transit Catalogue values were found to underestimate the total flux density for sources of LAS $\gtrsim 6^{\prime}$. For sources with a single MRC entry and LAS $<6^{\prime}$ the estimates were reliable. Of the 21 sources in this latter category, four were added to the sample.

After performing these various estimates of $S_{408}$, we were left with 20 extended MRC sources to add to the MS4 sample. They are listed in Table 2. Extended sources with integrated flux densities just below the 4.0 Jy cutoff are listed in Table 3.

\subsection{Completeness}

\subsubsection{Systematic Errors}

In spite of the work described in Section 2.3, the MS4 sample may still be incomplete, due to very extended sources. Since the MRC is complete only for sources with fitted flux density $>1 \mathrm{Jy}$, we may be missing some strong but diffuse sources with surface brightness $<1 \mathrm{Jy} /$ beam. However, such sources should have been catalogued in the lower resolution Parkes surveys, and a search in the region of interest for non-MRC sources with $S_{408}>4.0 \mathrm{Jy}$ located only three objects. Two of these, PKS B1209-52 and PKS B1210-52, are part of the Galactic supernova remnant G296.5+10.0 (Whiteoak \& Gardner 1968), and have not been included. The third, PKS B1400-33, is a low-surface-brightness possible relic radio source associated with the weak cluster around NGC 5419 (Goss et al. 1987; Subrahmanyan et al. 2003); this source was measured by SM75 and has been included in the MS4 sample.

The Parkes surveys did not have a uniform cutoff in Galactic latitude, and missed some regions with $|b|>10^{\circ}$. These excluded regions, however, occupy only a small fraction of the sky south of $-30^{\circ}$ (Ekers 1969a, Figure 1), and given the rarity of sources as diffuse as PKS B1400-33, it is unlikely that any other low-surface-brightness sources have been overlooked.

Completeness may be affected by the accidental omission of extended sources with components listed separately in the MRC. A giant source with compact lobes separated by $>8^{\prime}$ would have no extension flag in the MRC, and would not be in our list of extended candidates. There are several giant radio galaxies in Table 2, and we estimate that very few, if any, have been missed. 
Errors in estimating $S_{408}$ from the radio spectrum may affect completeness, particularly for values relying on extrapolation. The $843 \mathrm{MHz}$ integrated flux densities of JM92 were later found to be systematically low (see Section 3.4) by 6\%; this could, in principle, lead to an extrapolated $S_{408}$ for a genuine 4 Jy source being reduced below the sample limit. Extended sources falling just below the sample limit are listed in Table 3 .

\subsubsection{Random Errors}

The sample content may also be biased by random errors in the flux density measurements, as some sources with measured $S_{408}>4.0$ Jy may have true flux densities $<4.0$ Jy, and vice versa. Because the differential source counts have a negative slope near $4 \mathrm{Jy}$, slightly more sources will be wrongly included in the sample than will be wrongly excluded from it (Jauncey 1968). We estimated the number of sources likely to be affected by considering the flux densities and flux density errors of all MRC sources in the region of interest with measured flux densities between 1 and $10 \mathrm{Jy}$. Assuming the MRC flux-density errors were Gaussian, we calculated the expected number of sources with measured $S_{408}$ between 4 and $10 \mathrm{Jy}$ but true $S_{408}<4.0 \mathrm{Jy}$ to be 6.1 . Similarly we calculated the expected number of sources with measured $S_{408}$ between 1 and 4 Jy but true $S_{408}>4.0$ Jy to be 5.7.

The net number of sources wrongly included is therefore $<1$, so we can be confident that the sample size is not affected, although the sample content is not quite the same as it would be for zero measurement error. As the MRC errors are not actually Gaussian but have a long tail (Large et al. 1981), the numbers of sources thus brought into and taken out of the sample may be slightly larger than estimated here.

\subsubsection{Variability}

Flux-density variability can also cause a bias in sample content, but as the MS4 contains comparatively few flat-spectrum sources, only a small fraction of the sample can be affected by variability. While $30-50 \%$ of flat-spectrum sources are variable at $408 \mathrm{MHz}$ (Mantovani et al. 1992), less than $2 \%$ of steep-spectrum sources vary at low frequency (Dennison et al. 1981; Mantovani 1982). As only 14 of the $228 \mathrm{MS} 4$ sources have flat radio spectra ( $\alpha \geq-0.5$, defined in the sense $S_{\nu} \propto \nu^{\alpha}$ ), fewer than $5 \%$ of all MS4 sources are likely to vary significantly at $408 \mathrm{MHz}$. Consequently the effects of variability on sample size have been ignored. 


\subsubsection{Summary}

In summary, problems of incompleteness are likely to affect the net sample size only for extended sources. Fewer than five extended MRC sources are likely to have been wrongly excluded from the MS4 sample because of systematic underestimates of their flux densities. From examination of the lower resolution Parkes $408 \mathrm{MHz}$ surveys, few if any sources have been excluded through not being in the MRC. When the survey with the Mauritius radio telescope (Golap et al. 1998) at $151.5 \mathrm{MHz}$ has been analysed, it will provide an extra point in the radio spectrum to verify $S_{408}$ for extended sources north of $\delta=-70^{\circ}$.

\subsection{Comparison with the Northern Sample of Best et al. (1999)}

A sample similar to the MS4 has been defined from the MRC to have $S_{408}>5$ Jy in the declination range $-30^{\circ} \leq \delta \leq+10^{\circ}$ (Best et al. 1999, hereafter BRL99). Although containing useful radio and optical data, the BRL99 sample has the disadvantage - for

statistical studies - of radio incompleteness, as it was defined purely from the MRC flux densities, without examining those extended sources with MRC flux densities below 5 Jy.

To assess the number of sources likely to be missing from the BRL99 sample, we used the flux densities of the MS4 sample. In total, 160 MS4 sources had integrated $S_{408}>5$ Jy; of these 15 were extended sources with $S_{408}<5$ Jy in the MRC. The median angular size of these 15 sources was $450^{\prime \prime}$ and the minimum angular size was $126^{\prime \prime}$. These sources form around $10 \%$ of the MS4 sample with $S_{408}>5 \mathrm{Jy}$. We would expect a similar fraction to be missing from the BRL99 sample. The incompleteness is most likely to affect their sample at low redshifts, as they predict. We note that of the $15 \mathrm{MS} 4$ sources $\geq 5$ Jy which would have been excluded by the BRL99 selection criteria, 14 have $z<0.2$. This deficit is evident in the lowest redshift bin of Figure 53 of BRL99, and will necessarily compromise studies of the local radio luminosity function using this sample.

In assessing the completeness of their sample, BRL99 have misguidedly used the PKSCAT90 compilation (Otrupcek \& Wright 1991) rather than the original Parkes catalogues (Bolton et al. 1964; Shimmins et al. 1966; Day et al. 1966). Most of the $408 \mathrm{MHz}$ flux densities listed by PKSCAT90 are not Parkes measurements at all, but are taken directly from the MRC. This explains the large number of data points in Figure 52 of BRL99 for which exactly 100\% of the so-called "Parkes flux density" is contained in the MRC value. Unfortunately, there is no rationale given by the compilers of PKSCAT90 for when they chose to use MRC rather than Parkes data. The puzzling bimodal distribution in Figure 52 of BRL99 for angular sizes greater than $100^{\prime \prime}$ suggests that the decision was not related to angular size. As a result, 
flux densities, spectral indices, and radio powers for large angular size sources in Table 2 of BRL99 will be unreliable.

\subsection{The MS4 Sample}

The sample contains 228 sources, and is summarised in Table 4 . The columns of this table are as follows:

1. MRC name.

2. Parkes name. If there are two digits of declination this is from the original Parkes catalogue (Bolton et al. 1964; Price \& Milne 1965), and if three digits, from the Parkes $2700 \mathrm{MHz}$ catalogue (Bolton et al. 1979 and references therein).

3. Name of the source as given in the $85.5 \mathrm{MHz}$ MSH survey (Mills et al. 1960, 1961).

4. $408 \mathrm{MHz}$ flux density. This is usually from the MRC but for some extended sources is taken from other $408 \mathrm{MHz}$ measurements or estimated from the radio spectrum (see Section 2.3). References are given in Column 6 of Table 5 .

5. References to radio images in the literature, excluding VLBI images.

The sample covers an area of $2.43 \mathrm{sr}$, with a source density of $94 \mathrm{sr}^{-1}$, compared with the 3CRR sample which covers an area of $4.24 \mathrm{sr}$ and has a source density of $41 \mathrm{sr}^{-1}$. The higher source density is mainly due to the difference in flux-density cutoff. The 3CRR sample is defined by $S_{178} \geq 10.9 \mathrm{Jy}$, based on the flux-density scale of Baars et al. (1977) which agrees (at $408 \mathrm{MHz}$ ) with that of Wyllie (1969a) to $3 \%$. A source with $S_{178}=10.9$ Jy and spectral index of $\alpha=-0.81$, the median value for the 3CRR sample, will have $S_{408}=5.6 \mathrm{Jy}$. The sky distribution of the MS4 sources is shown in Figure 1.

The higher selection frequency means that the MS4 sample will have slightly different

properties from the 3CRR, containing more flat-spectrum sources. Because of the shape of the radio luminosity function, the lower flux-density cutoff means that the MS4 sample will contain a larger fraction of sources at high redshift.

\section{OBSERVATIONS AT $843 \mathrm{MHZ}$}

The sources have all been imaged with the Molonglo Observatory Synthesis Telescope (MOST) at $843 \mathrm{MHz}$. With better resolution and sensitivity than the $408 \mathrm{MHz}$ Molonglo 
Cross, the MOST provides improved information about source positions, structures, and blends. Flux densities at $843 \mathrm{MHz}$ conveniently bridge the gap between 408 and $1400 \mathrm{MHz}$. The positional accuracy of about $1^{\prime \prime}$ available with MOST makes it possible to obtain unambiguous optical identifications for most strong sources, even when the optical counterpart is faint.

The MOST is described by Mills (1981), Robertson (1991), and references therein. It was constructed from the $1.6 \mathrm{~km}$ east-west arm of the $408 \mathrm{MHz}$ Molonglo Cross Telescope, and modified to operate at $843 \mathrm{MHz}$ with a bandwidth of $3 \mathrm{MHz}$. Rather than recording complex visibilities, the MOST forms a comb of 128 fan beams in real time. An image of the sky is reconstructed from the fan-beam responses using back-projection (Perley 1979). The synthesised beam is $43^{\prime \prime} \times 43^{\prime \prime} \operatorname{cosec}|\delta|$, and the basic field size is $23^{\prime} \times 23^{\prime} \operatorname{cosec}|\delta|$; beam switching allows field sizes of up to $160^{\prime}$ to be observed (Bock et al. 1999). It requires twelve hours for full hour-angle coverage, in which case the $u v$ plane is totally filled within an ellipse, apart from a small hole at the centre corresponding to the $15 \mathrm{~m}$ gap between the East and West arms.

\section{1. 'CUTS' Observing}

Because of the large number of sources, the observations were done in 'CUTS' mode, a method of time-sharing in which about ten sources are observed in one 12-hour session. Most of the CUTS observing was performed by R.W.H. in 1985 and 1986, as part of a program to establish a grid of calibrators for MOST (Campbell-Wilson \& Hunstead 1994). Each source was observed in a cyclic schedule under computer control, with typical dwell times (CUTS) of 4 minutes at each of eight widely spaced hour angles. One or two unresolved sources were included as calibrators in each cycle.

In total 196 sources from the MS4 sample were observed in these CUTS runs, with the remainder being covered by full synthesis observations. Data reduction was performed using in-house software written by C. R. Subrahmanya, J. E. Reynolds, and T. Ye. The imaging process for CUTS is essentially the same as for full-synthesis data, but the software also allows calibration and viewing of individual CUTS, enabling angular sizes of compact sources to be estimated more accurately than from two-dimensional images. 


\subsection{Angular Sizes and Position Angles}

The signal in each CUT consists of a one-dimensional projection of the sky brightness distribution, convolved with the telescope's instantaneous point-spread function. Because they had higher angular resolution than the final two-dimensional images, the 1-D CUTS profiles were used to measure angular sizes and detect incipient double structure. The profiles were first deconvolved, using a 1-D CLEAN algorithm with a Gaussian restoring beam of FWHM 30". The dirty beam was estimated from the median of the CUTS profiles of the calibrators.

The largest angular extent and position angle of slightly resolved sources were determined by fitting a rectified sinewave to deconvolved source width as a function of hour angle. Comparison of repeat observations, and with higher-resolution ATCA images (Paper II), shows that for sources with LAS $>15^{\prime \prime}$, the position angles were accurate to about $5^{\circ}$, and the angular sizes to about $3^{\prime \prime}$.

For sources resolved into doubles we fitted a Gaussian to each component and measured the component separation directly. For sources with LAS $>1^{\prime}$ which were not edgebrightened doubles, we used the two-dimensional images to measure the angular size.

\subsection{Imaging and Deconvolution}

After removing individual CUTS affected by confusion in the fan beams, we formed raw two-dimensional images by back-projection. These images were then deconvolved with the standard CLEAN algorithm, using a dirty beam formed from the 1-D calibrator template. An example of raw and CLEANed CUTS images is shown in Hunstead (1991).

The dynamic range of the CLEANed images, defined as the ratio of the peak to the rms noise, was typically about 100:1, and was limited mainly by sidelobes from off-field sources, and by small variations in beam shape during the observation. For well resolved sources (LAS $\gtrsim 3^{\prime}$ ), the dynamic range was also limited by the success of CLEAN in modelling

extended structure. The dynamic range, although lower than available from a full-synthesis observation, was sufficient to define structure on arcminute angular scales, and to provide reliable positions.

Figure 2 contains MOST CUTS images of a selection of 12 MS4 sources which appear extended at $843 \mathrm{MHz}$ but which were not noted as extended in the MRC; all have angular extent $<2^{\prime}$. 


\subsection{Positions and Flux Densities}

Peak and centroid positions, and peak and integrated flux densities were measured from the CLEANed images. The peak positions and flux densities were obtained using a biquadratic fit to the pixel of highest flux density and the four pixels surrounding it.

\subsubsection{Flux Densities}

Integrated flux densities were obtained using the method of Jones (1989b). This involved considering the source brightness distribution as a surface in three-dimensional space, with the vertical axis proportional to flux density per beam area. The volume beneath this surface,

and above a given base level, was plotted as a function of base level and then extrapolated to a base level of zero. A zero base level was used because there was no evidence of a negative bowl - the absolute value of the mean flux density in the area surrounding the sources was well below the rms noise.

The flux-density scale used is that of Calabretta (1985), which was based on interpolation between $408 \mathrm{MHz}$ Molonglo and $2700 \mathrm{MHz}$ Parkes flux densities. Comparison of this scale with that of Caganoff (1984), based on absolute flux-density measurements at $843 \mathrm{MHz}$, yielded a mean flux-density ratio of $0.99 \pm 0.03$ for seven strong sources (Hunstead 1991). No northern hemisphere $843 \mathrm{MHz}$ measurements are available for further comparisons. We estimate the external accuracy of the flux-density calibration to be about $\pm 5 \%$. Based on repeated observations, we estimate the total flux-density error for compact sources (LAS $\left.\leq 1^{\prime}\right)$ to be $\pm 7 \%$ and for extended sources (LAS $>1^{\prime}$ ) to be about $\pm 10 \%$.

Comparison of integrated flux densities of 32 sources in the present study with the values reported by JM92 showed a significant systematic difference, with a mean ratio JM92/MS4 of $0.94 \pm 0.02$, with a standard deviation of 0.12 . The difference was significant at the $2 \%$ level, using a two-tailed Wilcoxon signed-rank test on the fractional difference in flux density. While the cause of this difference is unclear, the CUTS-based flux densities were preferred because they were more consistent with adjacent points in the radio spectra.

\subsubsection{Positions}

Position calibration of MOST is tied to a grid of unresolved sources (LAS $<10^{\prime \prime}$ ) with accurate centroid positions measured at $843 \mathrm{MHz}$ (Hunstead 1991). The calibrator errors are estimated to be $\sim 0 .^{\prime \prime} 2 \mathrm{rms}$ in right ascension and $\sim 0 .{ }^{\prime \prime} 2 \operatorname{cosec}|\delta| \mathrm{rms}$ in declination, 
with systematic errors $<0^{\prime \prime} .1$ (Campbell-Wilson \& Hunstead 1994).

We calculated the mean offsets in right ascension and declination for all the calibrators in each CUTS run, and used these to correct the measured target-source positions. The rms scatter was $1^{\prime \prime} .0$ in $\alpha$, and $1^{\prime \prime} .1 \operatorname{cosec}|\delta|$ in $\delta$. We also tested positional accuracy by comparing positions of 16 target sources in repeated observations. Median differences between pairs of positions for the 12 sources with LAS $<1^{\prime} .5$ were $0^{\prime \prime} .5$ in RA and $0^{\prime \prime} .9 \operatorname{cosec}|\delta|$ in Dec, and for the four sources with LAS $>1^{\prime} .5$, the median differences were $1^{\prime \prime} .5$ in RA and $1^{\prime \prime} .9 \operatorname{cosec}|\delta|$ in Dec. The error for extended sources is larger because of the uncertainties in determining the centroid at low flux-density levels.

The adopted position errors for the CUTS observations are therefore $\Delta \alpha=1^{\prime \prime} .0, \Delta \delta=$ $1^{\prime \prime} .1 \operatorname{cosec}|\delta|$ for sources with angular extent $<1^{\prime} .5$, and $\Delta \alpha=1^{\prime \prime} .5, \Delta \delta=1^{\prime \prime} .9 \operatorname{cosec}|\delta|$ for sources with angular extent $>1^{\prime} .5$.

\section{ESTIMATION OF FLUX DENSITIES AT $178 \mathrm{MHZ}$}

To compare the properties of the MS4 sources with those in the northern 3CRR sample, flux densities at $178 \mathrm{MHz}$ were estimated by interpolation or extrapolation from the radio spectrum. In Section 6, these flux densities will be used to define a southern 3CRR-equivalent sample.

For most sources the only flux-density measurements below $178 \mathrm{MHz}$ were those from the 85.5 MHz survey of Mills, Slee, and Hill (Mills et al. 1960, 1961), and those at 80 and $160 \mathrm{MHz}$ measured with the Culgoora radioheliograph (Slee \& Higgins 1973, Slee \& Higgins 1975 and Slee 1977; revised by Slee 1995). Because of their importance for interpolation, the $85.5 \mathrm{MHz} \mathrm{MSH}$ and $80 \mathrm{MHz}$ Culgoora values were compared to look for any systematic differences. The Culgoora flux densities, measured with a $3^{\prime} .7$ beam, were expected to be low for some extended sources, while the MSH values, measured with a $50^{\prime}$ beam, were likely to be overestimated due to confusion. It was therefore expected that the MSH values would be higher on average. If anything, the plot in Figure 3 shows that the opposite is true, although the scatter is large. Since the plot does not indicate a clear preference, both 80 and 85.5 MHz values were used in the fit (if available), unless other information led us to prefer one of the values.

Interpolation or extrapolation was done by performing a weighted least-squares polynomial fit to $\log S_{\nu}$ as a function of $\log \nu$. A quadratic fit was used in the first instance. If the reduced $\tilde{\chi}^{2}$ for the fit was greater than 5 , or if individual data points with small listed errors lay far from the fitted curve, a linear or cubic fit was attempted, depending on the apparent 
curvature of the spectrum and on the number of data points. Anomalous data points were excluded from the fit if there was evidence of confusion or over-resolution, or if the source was known to be variable. As the object was to optimise the fit at $178 \mathrm{MHz}$, high-frequency points $(\nu \geq 2700 \mathrm{MHz}$ ) were more likely to be excluded if they did not fit well with the low frequency spectrum.

Our estimates of $S_{178}$ are listed in Column 4 of Table 5; the order of the polynomial fit to $\log \nu$ is given to the right of the flux density. Comments are given below for sources in which the fit may be unreliable, based either on a large $\tilde{\chi}^{2}$ or on other information.

\subsection{Comments on Individual $178 \mathrm{MHz}$ Estimates}

MRC B0008-421: Required extrapolation below $408 \mathrm{MHz}$; estimated $S_{178}$ is unreliable as the radio spectrum is curved.

MRC B0240-422: The value of $S_{160}=11.5 \mathrm{Jy}$ is higher than expected from the fit; if correct, this source should be included in our southern equivalent of the 3CRR sample $(\S 6)$.

MRC B0438-436: Very compact source; the fit is probably affected by flux-density variability.

MRC B0454-463: Poor fit at low frequency $\left(\tilde{\chi}^{2}=11.5\right)$. A 5 GHz ATCA image (Paper 2) shows the radio structure to be core-dominated; the source is known to vary at high frequency (Wall et al. 1975; Wright et al. 1977).

MRC B0511-484: Most flux densities are affected by blending with two fainter sources; $S_{178}$ relies on extrapolation below $408 \mathrm{MHz}$.

MRC B0647-475: Compact source with a curved spectrum and flux densities at only four frequencies.

MRC B1315-460: Compact source with a curved spectrum and flux densities at only four frequencies.

PKS B1318-434: Flux densities are affected by confusion from Centaurus A, making the extrapolation to $178 \mathrm{MHz}$ unreliable. A quadratic fit to the four data points has been adopted; if the spectrum is really straight the source is probably stronger than the $3 \mathrm{CRR}$ cutoff of $S_{178}=10.9 \mathrm{Jy}$.

MRC B1445-468: The measurements at 80 and $85.5 \mathrm{MHz}$ are highly discrepant, so the fitted $S_{178}$ is unreliable. 
MRC B1545-321: Giant radio galaxy (LAS $=7^{\prime}$ ). The estimate of $S_{178}$ is unreliable as it relies on extrapolation from $843 \mathrm{MHz}$.

MRC B1549-790: Flat-spectrum source, known to vary at $843 \mathrm{MHz}$ (Gaensler \& Hunstead 2000); $S_{178}$ relies on extrapolation and may be unreliable.

MRC B1933-587: Poor fit to a polynomial at low frequency. A $5 \mathrm{GHz}$ ATCA image (Paper 2) shows the radio structure to be core-dominated; the source is known to vary at high frequency (Wall et al. 1975; Wright et al. 1977), but is non-variable at $843 \mathrm{MHz}$ (Gaensler \& Hunstead 2000).

MRC B1934-638: $S_{178}$ for this GPS source is very uncertain. We used the cubic polynomial fitted by Reynolds (1994).

MRC B2153-699: Extrapolation unreliable because of blending with MRC B2152-699.

\section{SUMMARY OF THE MS4 SAMPLE}

A summary of the $843 \mathrm{MHz}$ data for the MS4 sample, along with flux density measurements from the literature at other frequencies, is given in Table 5. Although different measurements in the literature have used (nominally) different flux-density scales, no correction has been made; the figures in the table are quoted directly from the papers cited. The columns of Table 5 are as follows:

1. Source name, taken from the MRC, unless the source has more than one MRC entry, in which case the name is from the original Parkes catalogue (Bolton et al. 1964; Price \& Milne 1965) or Parkes $2700 \mathrm{MHz}$ catalogue (Bolton et al. 1979 and references therein).

2. Right ascension in J2000 coordinates at $843 \mathrm{MHz}$.

3. Declination in J2000 coordinates at $843 \mathrm{MHz}$. The reference for the radio position in columns 2 and 3 is the same as for the $843 \mathrm{MHz}$ flux density in column 7 .

4. Estimated flux density at $178 \mathrm{MHz}$, as described in Section 4, and the order of the polynomial used to calculate the estimate.

5. Flux density at $80 \mathrm{MHz}$; the observing frequency is $85.5 \mathrm{MHz}$, if taken from $\mathrm{MSH}$, or $80 \mathrm{MHz}$, if measured with the Culgoora Circular Array. The latter flux densities are from the compilation of Slee (1995), with revised calibration. 
6. Flux density at $408 \mathrm{MHz}$, taken from the MRC, except for some extended sources. In general, flux densities were taken from published data in the following order of preference: SM75, MC4, Wyllie (1969b), the Parkes catalogues, Ekers et al. (1989). Flux densities for a few sources were estimated using the Molonglo Transit Catalogue or from the radio spectrum.

7. Flux density at $843 \mathrm{MHz}$, measured with MOST. The values of Campbell-Wilson \& Hunstead (1994) were used for MOST calibrators, as were those of Subrahmanyan et al. (1996) for six giant radio sources. For the remaining sources the flux density was taken either from JM92 or from the present study, depending on which image had the higher dynamic range.

8. Flux density at $1400 \mathrm{MHz}$. Data are mostly from Parkes (1410 MHz: Bolton et al. 1964; Price \& Milne 1965; Wills 1975), from Owens Valley (1425 MHz: Fomalont 1968; Fomalont \& Moffet 1971), or from the NRAO VLA Sky Survey catalog (NVSS; $1400 \mathrm{MHz}$ Condon et al. 1998). For a few sources data were taken from the Instituto Argentino de Radioastronomia (IAR) $30 \mathrm{~m}$ telescope (1410 MHz: Quiniento et al. 1988; Quiniento \& Echave 1990; Quiniento \& Cersosimo 1993), from the Fleurs Synthesis Telescope, or the Australia Telescope Compact Array. The flux-density scale for the Parkes catalogues (Bolton et al. 1964; Price \& Milne 1965) and Owens Valley data was that of Conway et al. (1963), whereas the data of Wills (1975) and the IAR $30 \mathrm{~m}$ data were on the scale of Wills (1973). At $1410 \mathrm{MHz}$ the scale of Wills (1973) is 1.08 times the scale of Conway et al. (1963) (Baars et al. 1977). NVSS used the flux density scale of Baars et al. (1977).

9. Flux density at $2700 \mathrm{MHz}$, measured with the Parkes telescope, except for one source which was observed at $2640 \mathrm{MHz}$ with Owens Valley (Rogstad \& Ekers 1969). $2650 \mathrm{MHz}$ flux densities are from the original Parkes catalogue or from Wills (1975), while $2700 \mathrm{MHz}$ values are from the Parkes $2700 \mathrm{MHz}$ survey or from Wills (1975). The Parkes $2700 \mathrm{MHz}$ survey used a scale in which the peak flux density of Hydra A was 23.5 Jy; Wills (1975) used the flux-density scale of Wills (1973).

10. Flux density at $5000 \mathrm{MHz}$, measured with the Parkes telescope. Values at $4850 \mathrm{MHz}$ are from the Parkes-MIT-NRAO survey (PMN: Gregory et al. 1994; Wright et al. 1996), those at $5000 \mathrm{MHz}$ are from Wills (1975), and those at $5009 \mathrm{MHz}$ are from Wills (1975), or the Parkes $2700 \mathrm{MHz}$ survey, or Shimmins et al. (1969), or Shimmins \& Bolton (1972a). The PMN survey used the flux density scale of Baars et al. (1977); Wills (1975) used the scale of Wills (1973); the Parkes $2700 \mathrm{MHz}$ surveys used a $5 \mathrm{GHz}$ scale in which the peak flux density of Hydra A was $S_{5009}=13.05 \mathrm{Jy}$, as did Shimmins et al. (1969) and Shimmins \& Bolton (1972a). 
11. Radio spectral index $\alpha$ measured between 408 and $2700 \mathrm{MHz}$, defined in the sense $S_{\nu} \propto \nu^{\alpha}$. For sources without measurements at both those frequencies, the spectral index was measured either between 408 and $5000 \mathrm{MHz}$ or between 843 and $5000 \mathrm{MHz}$.

12. Largest angular size (LAS) at $843 \mathrm{MHz}$. The reference is the same as for the $843 \mathrm{MHz}$ flux density, except for a few sources, noted in Section 5.1. We have imaged all sources with LAS $<35^{\prime \prime}$ with the ATCA at $5 \mathrm{GHz}$ (Paper II), with the exception of MRC B0521-365, MRC B0743-673, MRC B1740-517, MRC B1814-519, MRC B2153-699, and MRC B2259-375.

13. Position angle of extension at $843 \mathrm{MHz}$, defined in degrees east of north (modulo 90). The reference is the same as for the $843 \mathrm{MHz}$ flux density, except for a few sources which are noted in Section 5.1.

\subsection{Comments on Individual Sources}

MRC B0208-512: Detected with EGRET (Thompson et al. 1995).

MRC B0214-480: The Parkes 408 MHz and MSH 85.5 MHz flux densities are probably affected by blending with MRC B0211-479. The position, angular size, and position angle in Table 5 are from JM92, but $S_{843}$ was measured from MOST images from the SUMSS survey (Mauch et al. 2003).

MRC B0252-712: The spectrum turns over at low frequency. The flux density of $S_{85.5}=7 \mathrm{Jy}$ is noted as uncertain in $\mathrm{MSH}$, but corresponds well with the slight spectral curvature evident at higher frequencies.

PKS B0319-45: Giant radio galaxy. Because of the large angular size, the flux densities are uncertain. The MSH $85.5 \mathrm{MHz}$ measurement was affected by sidelobes of Fornax A.

MRC B0320-373: Fornax A.

MRC B0336-355: Blended at low resolution with the foreground source MRC B0336-356. At 2650 and $5009 \mathrm{MHz}$, the peak flux densities (Wills 1975) were used, as they are expected to be less affected by blending.

MRC B0411-346A: The weaker source MRC B0411-346B is unrelated.

MRC B0453-301, MRC B0456-301: MSH 04-314 is a blend of these two sources.

MRC B0506-612: The 8.87 GHz flux density of 2.63 Jy (Shimmins \& Wall 1973) is substantially higher than expected from the radio spectrum, probably indicating high-frequency 
variability.

MRC B0511-484: Unequal double, with two neighbouring weaker sources, presumed to be unrelated. The SM75 integrated value of $S_{408}=8.8 \mathrm{Jy}$ is affected by blending. The MRC fitted value of $6.84 \mathrm{Jy}$ was preferred but is still uncertain. The Parkes flux densities at 2.7 and $5 \mathrm{GHz}$ are also affected by blending, so the radio spectrum is not well determined.

MRC B0518-458: Pictor A.

MRC B0521-365: Well studied blazar, detected as a gamma-ray source by EGRET (Thompson et al. 1995).

PKS B0707-35: The flux density $S_{5000}=0.84$ Jy (Wall \& Schilizzi 1979) appears anomalously high.

MRC B0743-673: The $1410 \mathrm{MHz}$ flux density (Price \& Milne 1965) is higher than expected from the radio spectrum, and probably affected by blending with MRC B0742-674.

MRC B1017-421, MRC B1017-426: MSH 10-44 is a blend of these two sources.

MRC B1136-320: In the Texas catalog (Douglas et al. 1996) the double separation is quoted as $58^{\prime \prime}$ in P.A. $-15^{\circ}$, consistent with the values in Table 5.

MRC B1143-316: The Texas catalog (Douglas et al. 1996) lists this source as a double with separation $34^{\prime \prime}$ in P.A. $86^{\circ}$. The MRC value of $S_{408}=5.77 \mathrm{Jy}$ is preferred to SM75's anomalously low value of $3.8 \mathrm{Jy}$.

MRC B1302-491: Nearby edge-on spiral galaxy NGC 4945, identified by Mills et al. (1960).

PKS B1318-434: Complex double source, with bent edge-darkened lobes, lying behind the southern lobe of Centaurus A (Cooper et al. 1965; Haynes et al. 1983).

MRC B1322-427: The well studied low-luminosity radio galaxy Centaurus A (see review by Ebneter \& Balick 1983). The flux density at $843 \mathrm{MHz}$ was determined from the radio spectrum.

PKS B1400-33: The only source in the sample which is not in the MRC. An unusual extended source of low surface brightness. There is a nearby compact source associated with the E0 galaxy NGC 5419 (see Ekers et al. 1989). Goss et al. (1987) suggest that the extended component may be a relic radio source associated with the poor cluster Abell S753 around NGC 5419. The radio properties have been studied in detail by Subrahmanyan et al. (2003); we have used their flux densities at 843 and $1398 \mathrm{MHz}$. 
MRC B1425-479: Because of the angular size of $4^{\prime} .5$, the values of $S_{408}$ and $S_{5000}$ in Table 5 may be slightly underestimated.

MRC B1445-468: The published values of $S_{80}-2 \mathrm{Jy}$ (Slee 1995) and $13 \mathrm{Jy}$ (Slee \& Higgins 1973) — are grossly discrepant. We prefer the latter value, after multiplying it by 1.1 to correct the flux-density scale (Slee 1977).

MRC B1549-790: Flat-spectrum source. The flux density at $1410 \mathrm{MHz}$ (Price \& Milne $1965)$ is affected by blending with MRC B1547-795.

MRC B1814-637, MRC B1817-640: MSH 18-61 is a blend of these two sources.

MRC B1917-546: Ultra-steep spectrum source. The $1410 \mathrm{MHz}$ flux density may be affected by blending with two weak neighbouring sources (Hunstead 1972).

MRC B1934-638: Archetypal Gigahertz-Peaked-Spectrum (GPS) source (Bolton et al. 1963).

MRC B1940-406: Blending with three weaker sources (JM92) makes the radio spectrum uncertain.

MRC B2006-566: Very extended, diffuse source, associated with the cluster Abell 3667 (Abell et al. 1989). The flux density of $S_{843}=(5.5 \pm 0.5)$ Jy (Röttgering et al. 1997) was obtained from a MOST full-synthesis image after subtracting the contribution from background sources within the source envelope (0.6 Jy); the discrepancy with JM92 is probably due to the higher noise level in their image.

MRC B2052-474: Detected with EGRET (Thompson et al. 1995).

MRC B2122-555: The MRC position appears to be in error, lying $30^{\prime \prime}$ east of the MOST position. The MRC flux density of 4.05 Jy is also lower than expected from the radio spectrum, suggesting that the observation may have been affected by a large ionospheric wedge (Hunstead 1972). Both Parkes positions (Wall et al. 1975; Gregory et al. 1994) agree with the MOST centroid.

MRC B2152-699: Some flux densities are affected by blending with MRC B2153-699. The $1415 \mathrm{MHz}$ measurement (Christiansen et al. 1977) was made with the Fleurs Synthesis Telescope. At $2650 \mathrm{MHz}$, the peak flux density (Wills 1975) was used, to reduce any contribution from MRC B2153-699.

MRC B2153-699: Double, extended along a position angle similar to that of MRC B2152-699. Flux densities are affected by blending with MRC B2152-699: both the MRC value of $20.9 \pm 1.4 \mathrm{Jy}$ and the SM75 value of $6.0 \pm 0.4 \mathrm{Jy}$ are regarded as unreliable. Therefore, the 
flux densities at 843 and $1415 \mathrm{MHz}$, together with the $468 \mathrm{MHz}$ value of $10.7 \pm 0.9 \mathrm{Jy}$ (Ekers 1969b), have been used to estimate the flux density at $408 \mathrm{MHz}$.

\section{DISCUSSION}

The $178 \mathrm{MHz}$ flux densities estimated in Section 4 have been used to define a strong source subsample of MS4 which we call SMS4. It has been chosen to have the same fluxdensity cutoff, $10.9 \mathrm{Jy}$, as the northern 3CRR sample, and contains 137 sources, compared with 172 in 3 CRR.

Comparison of SMS4 with 3CRR (Table 6) shows the southern sample to have a slightly higher source density, but only at the $2.8 \sigma$ level of significance. The difference should be treated with caution and may simply reflect biases in the way each sample was compiled. We have identified three possible causes of such a bias:

(i) As the spectra of many radio sources turn over at low radio frequency, extrapolation from high frequencies is more likely to overestimate than underestimate $S_{178}$. The survey with the Mauritius radio telescope (Golap et al. 1998) at $151.5 \mathrm{MHz}$ will be valuable for checking flux densities of sources north of $\delta=-70^{\circ}$, and testing for such a bias in the SMS4 sample.

(ii) Because of the steep slope of the radio source counts, a small systematic difference in flux-density scale can strongly bias the source density. The Mauritius values will be useful for checking such effects in SMS4.

(iii) The 3CRR may be missing sources of low surface brightness. The angular size distributions are compared in Figure 4. The median angular size of SMS4 is $32_{-5}^{+6}$ arcsec, compared with $35.5_{-7.5}^{+8.7}$ arcsec for 3CRR. Although the medians are similar, the distributions appear different: the SMS4 has proportionally more sources with angular size $>300^{\prime \prime}$, and proportionally fewer sources with angular size between 100" and 300". However, because of the small numbers of sources involved, it is not possible to draw firm conclusions about differences in the angular size distributions.

The median flux density of SMS4 $\left(S_{178}=15.7 \mathrm{Jy}\right)$, is consistent with the median value of 15.6 Jy for 3CRR. This is to be expected if the radio source counts are similar. Histograms of spectral indices for the MS4, SMS4, and 3CRR samples are plotted in Figure 5. The distributions for the MS4 sample show a longer tail towards flatter spectral indices than do those of the SMS4 or 3CRR. This is to be expected given the higher selection frequency 
of the MS4, as flat-spectrum sources will be included which would be missed with a higher flux-density cutoff and lower selection frequency. The median spectral indices, $\alpha=-0.91$ for SMS4 and -0.81 for 3CRR (Table 6), are not consistent, but can be explained simply by the different frequency ranges used to measure $\alpha$ for each sample: $408-2700 \mathrm{MHz}$ for SMS4 and 178-750 MHz for 3CRR. Given that the spectra of many radio sources turn over at low frequency, and that radio spectra often steepen at high frequency, the flatter median spectral index of the 3CRR sources can be explained by the lower frequency range over which $\alpha$ was measured. A comparison over more similar frequency ranges will be possible when 151.5 MHz data from Mauritius become available.

\section{SUMMARY}

The Molonglo Southern $4 \mathrm{Jy}$ (MS4) sample, a complete sample of radio sources south of $\delta=-30^{\circ}$, has been selected from the Molonglo Reference Catalogue (MRC) to have $S_{408}>4.0 \mathrm{Jy}$. All the MS4 sources have been imaged with MOST at $843 \mathrm{MHz}$, thus providing more accurate positions than available from the MRC. The positional accuracy is about $\Delta \alpha=$ $1^{\prime \prime} .0, \Delta \delta=1^{\prime \prime} .1 \operatorname{cosec}|\delta|$ for compact sources (angular size $<1^{\prime} .5$ ), and $\Delta \alpha=1^{\prime \prime} .5, \Delta \delta=$ $1^{\prime \prime} .9 \operatorname{cosec}|\delta|$ for extended sources (angular size $>1^{\prime} .5$ ). Integrated flux densities at $843 \mathrm{MHz}$ have been measured from the images, and have an accuracy of $\sim 7-10 \%$. Reliable angular

sizes and position angles have been measured for sources with angular extent $\gtrsim 15^{\prime \prime}$. The median angular size for the MS4 sample as measured with MOST is $23^{\prime \prime} \pm 4^{\prime \prime}$.

The set of MOST images provides a useful database for optical identification, as well as for planning higher-resolution radio imaging, to be presented in Paper II. Radio flux densities at other frequencies have been obtained from the literature, and used to retrospectively define a stronger subsample, the SMS4, with the same flux-density limit as the $178 \mathrm{MHz} 3 \mathrm{CRR}$ sample. Preliminary comparisons of SMS4 and 3CRR show that their overall properties are similar.

\section{ACKNOWLEDGEMENTS}

We thank staff and students of the School of Physics, University of Sydney, for their generous help with observing and data reduction. Special thanks to the staff of the Molonglo Telescope, for operating and maintaining it for the demanding CUTS runs, and to T. Ye and C. R. Subrahmanya for writing and modifying data-reduction software.

We have made use of NASA's Astrophysics Data System Abstract Service, and the 
NASA/IPAC Extragalactic Database (NED) which is operated by the Jet Propulsion Laboratory, Caltech, under contract with the US National Aeronautics and Space Administration. The Molonglo Observatory Synthesis Telescope is funded by both the Australian Research Council and the Science Foundation for Physics within the University of Sydney. AMB acknowledges the receipt of an Australian Postgraduate Research Award over the period of this research. 


\section{REFERENCES}

Abell, G. O., Corwin, H. G., \& Olowin, R. P. 1989, ApJS, 70, 1

Alvarez, H., Aparici, J., May, J., \& Reich, P. 2000, A\&A, 355, 863

Baars, J. W. M., Genzel, R., Pauliny-Toth, I. I. K., \& Witzel, A. 1977, A\&A, 61, 99

Bennett, A. S. 1962, Mem. R. Astron. Soc., 68, 163

Best, P. N., Röttgering, H. J. A., \& Lehnert, M. D. 1999, MNRAS, 310, 223, arXiv:astroph/9903471 (BRL99)

Birkinshaw, M., Worrall, D. M., \& Hardcastle, M. J. 2002, MNRAS, 335, 142, arXiv:astro$\mathrm{ph} / 0204509$

Bock, D. C.-J., Large, M. I., \& Sadler, E. M. 1999, AJ, 117, 1578, arXiv:astro-ph/9812083

Bolton, J. G. \& Butler, P. W. 1975, Aust. J. Phys. Astrophys. Suppl., 34, 33

Bolton, J. G. \& Clark, B. G. 1960, PASP, 72, 29

Bolton, J. G., Gardner, F. F., \& Mackey, M. B. 1963, Nature, 199, 682

—. 1964, Aust. J. Phys., 17, 340

Bolton, J. G. \& Shimmins, A. J. 1973, Aust. J. Phys. Astrophys. Suppl., 30, 1

Bolton, J. G., Wright, A. E., \& Savage, A. 1979, Aust. J. Phys. Astrophys. Suppl., 46, 1

Bosma, A., Goss, W. M., \& Wellington, K. J. 1983, A\&AS, 54, 387

Burgess, A. M. \& Hunstead, R. W. 2006, AJ, in press (Paper II)

Burns, J. O., Feigelson, E. D., \& Schreier, E. J. 1983, ApJ, 273, 128

Caganoff, S. 1984, Physics IV Honours Report, University of Sydney

Calabretta, M. R. 1985, PhD thesis, University of Sydney

Cameron, M. J. 1971, MNRAS, 152, 439

Campbell-Wilson, D. \& Hunstead, R. W. 1994, Proc. Astron. Soc. Aust., 11, 33

Carter, D. \& Malin, D. F. 1983, MNRAS, 203, 49P

Chartas et al. 2000, ApJ, 542, 655, arXiv:astro-ph/0005227 
Choi, Y., Reynolds, C. S., Heinz, S., Rosenberg, J. L., Perlman, E. S., \& Yang, J. 2004, ApJ, 606, 185, arXiv:astro-ph/0402131

Christiansen, W. N., Frater, R. H., Watkinson, A., O’Sullivan, J. D., Lockhart, I. A., \& Goss, W. M. 1977, MNRAS, 181, 183

Clark et al. 1997, MNRAS, 286, 558

Clarke, D. A., Burns, J. O., \& Norman, M. L. 1992, ApJ, 395, 444

Clarke, J. N., Little, A. G., \& Mills, B. Y. 1976, Aust. J. Phys. Astrophys. Suppl., 40, 1 (MC4)

Combi, J. A. \& Romero, G. E. 1997, A\&AS, 121, 11

Condon, J. J., Cotton, W. D., Greisen, E. W., Yin, Q. F., Perley, R. A., Taylor, G. B., \& Broderick, J. J. 1998, AJ, 115, 1693

Condon, J. J., Helou, G., Sanders, D. B., \& Soifer, B. T. 1996, ApJS, 103, 81

Conway, R. G., Kellermann, K. I., \& Long, R. J. 1963, MNRAS, 125, 261

Cooper, B. F. C., Price, R. M., \& Cole, D. J. 1965, Aust. J. Phys., 18, 589

Dahlem, M., Golla, G., Whiteoak, J. B., Wielebinski, R., Huttemeister, S., \& Henkel, C. 1993, A\&A, 270, 29

Danziger, I. J. \& Goss, W. M. 1983, MNRAS, 202, 703

Day, G. A., Shimmins, A. J., Ekers, R. D., \& Cole, D. J. 1966, Aust. J. Phys., 19, 35

De Breuck, C., Hunstead, R. W., Sadler, E. M., Rocca-Volmerange, B., \& Klamer, I. 2004, MNRAS, 347, 837, arXiv:astro-ph/0309814

De Breuck, C., van Breugel, W., Röttgering, H. J. A., \& Miley, G. 2000, A\&AS, 143, 303, arXiv:astro-ph/0002297

de Pater, I., Schloerb, F. P., \& Johnson, A. H. 1985, AJ, 90, 846

Dennison, B., Broderick, J. J., Ledden, J. E., \& O’Dell, S. L. 1981, AJ, 86, 1604

Douglas, J. N., Bash, F. N., Bozyan, F. A., Torrence, G. W., \& Wolfe, C. 1996, AJ, 111, 1945

Drinkwater et al. 1997, MNRAS, 284, 85, arXiv:astro-ph/9609019 
Duncan, R. A. \& Sproats, L. N. 1992, Proc. Astron. Soc. Aust., 10, 16

Ebneter, K. \& Balick, B. 1983, PASP, 95, 675

Edge, D. O., Shakeshaft, J. R., McAdam, W. B., Baldwin, J. E., \& Archer, S. 1959, Mem. R. Astron. Soc., 68, 37

Ekers, J. A. 1969a, Aust. J. Phys. Astrophys. Suppl., 7, 1

Ekers, R. D. 1969b, Aust. J. Phys. Astrophys. Suppl., 6, 1

Ekers, R. D., Goss, W. M., Kotanyi, C. G., \& Skellern, D. J. 1978, A\&A, 69, L21

Ekers, R. D., Goss, W. M., Wellington, K. J., Bosma, A., Smith, R. M., \& Schweizer, F. 1983, A\&A, 127, 361

Ekers, R. D. \& Whiteoak, J. B. 1992, Journal of Electrical and Electronics Engineering, Australia, 12, 225

Ekers et al. 1989, MNRAS, 236, 737

Elmouttie, M., Haynes, R. F., Jones, K. L., Ehle, M., Beck, R., Harnett, J. I., \& Wielebinski, R. 1997, MNRAS, 284, 830

Fomalont, E. B. 1968, ApJS, 15, 203

Fomalont, E. B., Ebneter, K. A., van Breugel, W. J. M., \& Ekers, R. D. 1989, ApJ, 346, L17

Fomalont, E. B. \& Moffet, A. T. 1971, AJ, 76, 5

Forbes, D. A. \& Norris, R. P. 1998, MNRAS, 300, 757, arXiv:astro-ph/9804298

Fosbury, R. A. E., Morganti, R., Wilson, W., Ekers, R. D., di Serego Alighieri, S., \& Tadhunter, C. N. 1998, MNRAS, 296, 701, arXiv:astro-ph/9801249

Fosbury et al. 1982, MNRAS, 201, 991

Gaensler, B. M. \& Hunstead, R. W. 2000, Publ. Astron. Soc. Aust., 17, 72, arXiv:astro$\mathrm{ph} / 9911194$

Gardner, F. F. \& Whiteoak, J. B. 1971, Aust. J. Phys., 24, 899

Gardner, F. F., Whiteoak, J. B., \& Morris, D. 1969, Aust. J. Phys., 22, 821

Geldzahler, B. J. \& Fomalont, E. B. 1984, AJ, 89, 1650 
Gliozzi, M., Brinkmann, W., Laurent-Muehleisen, S. A., Takalo, L. O., \& Sillanpää, A. 1999, A\&A, 352, 437, arXiv:astro-ph/9911055

Golap, K., Udaya Shankar, N., Sachdev, S., Dodson, R., \& Sastry, C. V. 1998, JA\&A, 19, 35, arXiv:astro-ph/9808062

Goldstein, S. J. 1962, AJ, 67, 171

Goss, W. M., Ekers, R. D., Skellern, D. J., \& Smith, R. M. 1982, MNRAS, 198, 259

Goss, W. M., McAdam, W. B., Wellington, K. J., \& Ekers, R. D. 1987, MNRAS, 226, 979

Gregorini, L., de Ruiter, H. R., Parma, P., Sadler, E. M., Vettolani, G., \& Ekers, R. D. 1994, A\&AS, 106, 1

Gregory, P. C., Vavasour, J. D., Scott, W. K., \& Condon, J. J. 1994, ApJS, 90, 173

Griffith, M. R., Wright, A. E., Burke, B. F., \& Ekers, R. D. 1994, ApJS, 90, 179

-. 1995, ApJS, 97, 347

Haigh, A. D. 2000, PhD thesis, University of Sydney

Hardcastle, M. J., Worrall, D. M., \& Birkinshaw, M. 1999, MNRAS, 305, 246, arXiv:astro$\mathrm{ph} / 9812148$

Hardcastle, M. J., Worrall, D. M., Kraft, R. P., Forman, W. R., Jones, C., \& Murray, S. S. 2003, ApJ, 593, 169, arXiv:astro-ph/0304443

Harnett, J. I. 1987, MNRAS, 227, 887

Harnett, J. I., Haynes, R. F., Klein, U., \& Wielebinski, R. 1989, A\&A, 216, 39

Harnett, J. I., Haynes, R. F., Wielebinski, R., \& Klein, U. 1990, Proc. Astron. Soc. Aust., 8,257

Harnett, J. I. \& Reynolds, J. E. 1985, MNRAS, 215, 247

Haslam et al. 1981, A\&A, 100, 209

Haynes, R. F., Cannon, R. D., \& Ekers, R. D. 1983, Proc. Astron. Soc. Aust., 5, 241

Heeschen, D. S. 1961, ApJ, 133, 322

Heinz, S., Choi, Y., Reynolds, C. S., \& Begelman, M. C. 2002, ApJ, 569, L79, arXiv:astroph/0201107 
Hunstead, R. W. 1972, MNRAS, 157, 367

—. 1991, Aust. J. Phys., 44, 743

Hunstead, R. W., Durdin, J. M., Little, A. G., Reynolds, J. E., \& Kesteven, M. J. 1982, Proc. Astron. Soc. Aust., 4, 447

Jauncey, D. L. 1968, ApJ, 152, 647

Jones, P. A. 1989a, Proc. Astron. Soc. Aust., 8, 81

—. 1989b, PhD thesis, University of Sydney

—. 1992, Aust. J. Phys., 45, 797

Jones, P. A. \& McAdam, W. B. 1992, ApJS, 80, 137 (JM92)

Junkes, N., Haynes, R. F., Harnett, J. I., \& Jauncey, D. L. 1993a, A\&A, 269, 29

-. 1993b, A\&A, 274, 1009

Kaufmann, P., Scalise, E., Marques dos Santos, P., \& Fogarty, W. G. 1974, MNRAS, 169, $15 \mathrm{P}$

Keel, W. C. 1986, ApJ, 302, 296

Killeen, N. E., Bicknell, G. V., \& Ekers, R. D. 1986, ApJ, 302, 306

Killeen, N. E. B. \& Bicknell, G. V. 1988, ApJ, 325, 180

Kraft, R. P., Forman, W. R., Jones, C., Murray, S. S., Hardcastle, M. J., \& Worrall, D. M. 2002, ApJ, 569, 54, arXiv:astro-ph/0111340

Kraft et al. 2003, ApJ, 592, 129, arXiv:astro-ph/0304363

Laing, R. A., Riley, J. M., \& Longair, M. S. 1983, MNRAS, 204, 151

Large, M. I., Mills, B. Y., Little, A. G., Crawford, D. F., \& Sutton, J. M. 1981, MNRAS, 194, 693

Lloyd, B. D. \& Jones, P. A. 2002, MNRAS, 331, 717

Lloyd, B. D., Jones, P. A., \& Haynes, R. F. 1996, MNRAS, 279, 1197

Lockhart, I. A. \& Sheridan, K. V. 1970, Proc. Astron. Soc. Aust., 1, 344 
Mantovani, F. 1982, A\&A, 110, 345

Mantovani, F., Junor, W., Fanti, R., Padrielli, L., Browne, I. W. A., \& Muxlow, T. W. B. 1992, MNRAS, 257, 353

Marvel, K. B., Shukla, H., \& Rhee, G. 1999, ApJS, 120, 147

Mauch et al. 2003, MNRAS, 342, 1117

McAdam, W. B. \& Schilizzi, R. T. 1977, A\&A, 55, 67

McAdam, W. B., White, G. L., \& Bunton, J. D. 1988, MNRAS, 235, 425

McGee, R. X., Slee, O. B., \& Stanley, G. J. 1955, Aust. J. Phys., 8, 347

Meisenheimer, K., Röser, H.-J., Hiltner, P. R., Yates, M. G., Longair, M. S., Chini, R., \& Perley, R. A. 1989, A\&A, 219, 63

Mills, B. Y. 1981, Proc. Astron. Soc. Aust., 4, 156

Mills, B. Y., Aitchison, R. E., Little, A. G., \& McAdam, W. B. 1963, Proc. Inst. Radio Engrs. Aust., 24, 156

Mills, B. Y., Slee, O. B., \& Hill, E. R. 1958, Aust. J. Phys., 11, 360

—. 1960, Aust. J. Phys., 13, 676

—. 1961, Aust. J. Phys., 14, 497

Morganti, R., Killeen, N. E. B., Ekers, R. D., \& Oosterloo, T. A. 1999a, MNRAS, 307, 750, arXiv:astro-ph/9903448

Morganti, R., Killeen, N. E. B., \& Tadhunter, C. N. 1993, MNRAS, 263, 1023

Morganti, R., Oosterloo, T., Tadhunter, C. N., Aiudi, R., Jones, P., \& Villar-Martin, M. 1999b, A\&AS, 140, 355, arXiv:astro-ph/9910150

Morganti, R., Oosterloo, T. A., Tadhunter, C. N., van Moorsel, G., Killeen, N., \& Wills, K. A. 2001, MNRAS, 323, 331, arXiv:astro-ph/0010636

Norris et al. 1990, Proc. Astron. Soc. Aust., 8, 252

O’Dea, C. P., Baum, S. A., Maloney, P. R., Tacconi, L. J., \& Sparks, W. B. 1994, ApJ, 422, 467 
Otani, C., Brinkmann, W., Böhringer, H., Reid, A., \& Siebert, J. 1998, A\&A, 339, 693

Otrupcek, R. E. \& Wright, A. E. 1991, Proc. Astron. Soc. Aust., 9, 170

Ott, M., Whiteoak, J. B., Henkel, C., \& Wielebinski, R. 2001, A\&A, 372, 463, arXiv:astro$\mathrm{ph} / 0109190$

Parma, P., Cameron, R. A., \& Deruitter, H. R. 1991, AJ, 102, 1960

Perley, R. A. 1979, AJ, 84, 1443

Perley, R. A., Röser, H.-J., \& Meisenheimer, K. 1997, A\&A, 328, 12

Price, R. M. \& Milne, D. K. 1965, Aust. J. Phys., 18, 329

Quiniento, Z. M. \& Cersosimo, J. C. 1993, A\&AS, 97, 435

Quiniento, Z. M., Cersosimo, J. C., \& Colomb, F. R. 1988, A\&AS, 76, 21

Quiniento, Z. M. \& Echave, M. M. 1990, A\&AS, 83, 393

Rayner, D. P., Norris, R. P., \& Sault, R. J. 2000, MNRAS, 319, 484

Reid, R. I., Kronberg, P. P., \& Perley, R. A. 1999, ApJS, 124, 285

Reynolds, J. 1994, Flux Scale for the AT Compact Array, Tech. Rep. 39.3/040, ATNF

Reynolds et al. 1993, in Sub-arcsecond Radio Astronomy, ed. R. J. Davis \& R. S. Booth (Cambridge: Cambridge University Press), 156

Robertson, J. G. 1991, Aust. J. Phys., 44, 729

Robertson, J. G. \& Smith, R. M. 1981, Proc. Astron. Soc. Aust., 4, 187

Rogstad, D. H. \& Ekers, R. D. 1969, ApJ, 157, 481

Röttgering, H. J. A., Wieringa, M. H., Hunstead, R. W., \& Ekers, R. D. 1997, MNRAS, 290, 577

Sadler, E. M. 1987, in Observational Evidence of Activity in Galaxies, ed. E. Y. Khachikian, K. J. Fricke, \& J. Melnick, IAU Symp. 121 (Dordrecht: Reidel), 443

Sadler, E. M. 1997, in ASP Conf. Ser., Vol. 116, The Second Stromlo Symposium: The Nature of Elliptical Galaxies, ed. M. Arnaboldi, G. S. Da Costa, \& P. Saha (San Francisco: ASP), 411 
Safouris, V., Hunstead, R. W., \& Prouton, O. R. 2003, Publ. Astron. Soc. Aust., 20, 1

Saripalli, L., Subrahmanyan, R., \& Hunstead, R. W. 1994, MNRAS, 269, 37

Saripalli, L., Subrahmanyan, R., \& Udaya Shankar, N. 2002, ApJ, 565, 256, arXiv:astro$\mathrm{ph} / 0209570$

-. 2003, ApJ, 590, 181, arXiv:astro-ph/0303157

Sarma, A. P., Troland, T. H., \& Rupen, M. P. 2002, ApJ, 564, 696

Sault, R. J. \& Wieringa, M. H. 1994, A\&AS, 108, 585

Schilizzi, R. T. \& McAdam, W. B. 1975, Mem. R. Astron. Soc., 79, 1 (SM75)

Schiminovich, D., van Gorkom, J. H., van der Hulst, J. M., \& Kasow, S. 1994, ApJ, 423, L101

Schreier, E. J., Burns, J. O., \& Feigelson, E. D. 1981, ApJ, 251, 523

Schwartz, D. A., Bradt, H. V., Remillard, R. A., \& Tuohy, I. R. 1991, ApJ, 376, 424

Schwartz et al. 2000, ApJ, 540, L69, arXiv:astro-ph/0005255

Schwarz, U. J., Cole, D. J., \& Morris, D. 1973, Aust. J. Phys., 26, 661

Schwarz, U. J., Whiteoak, J. B., \& Cole, D. J. 1974, Aust. J. Phys., 27, 563

Shain, C. A. 1958, Aust. J. Phys., 11, 517

Sheridan, K. V. 1958, Aust. J. Phys., 11, 400

Shimmins, A. J. 1971, Aust. J. Phys. Astrophys. Suppl., 21, 1

Shimmins, A. J. \& Bolton, J. G. 1972a, Aust. J. Phys. Astrophys. Suppl., 23, 1

—. 1972b, Aust. J. Phys. Astrophys. Suppl., 26, 1

—. 1974, Aust. J. Phys. Astrophys. Suppl., 32, 1

Shimmins, A. J. \& Day, G. A. 1968, Aust. J. Phys., 21, 377

Shimmins, A. J., Day, G. A., Ekers, R. D., \& Cole, D. J. 1966, Aust. J. Phys., 19, 837

Shimmins, A. J., Manchester, R. N., \& Harris, B. J. 1969, Aust. J. Phys. Astrophys. Suppl., 8,1 
Shimmins, A. J. \& Wall, J. V. 1973, Aust. J. Phys. Astrophys. Suppl., 26, 93

Simkin, S. M., Sadler, E. M., Sault, R., Tingay, S. J., \& Callcut, J. 1999, ApJS, 123, 447, arXiv:astro-ph/9903313

Slee, O. B. 1977, Aust. J. Phys. Astrophys. Suppl., 43, 1

—. 1995, Aust. J. Phys., 48, 143

Slee, O. B. \& Higgins, C. S. 1973, Aust. J. Phys. Astrophys. Suppl., 27, 1

—. 1975, Aust. J. Phys. Astrophys. Suppl., 36, 1

Slee, O. B. \& Sheridan, K. K. 1975, Proc. Astron. Soc. Aust., 2, 336

Slee, O. B., Sheridan, K. V., Dulk, G. A., \& Little, A. G. 1983, Proc. Astron. Soc. Aust., 5, 247

Smith, R. M. 1983, PhD thesis, Australian National University

Smith, R. M. \& Bicknell, G. V. 1983, Proc. Astron. Soc. Aust., 5, 251

—. 1986, ApJ, 308, 36

Stephenson, F., Clark, D. H., \& Crawford, D. F. 1977, MNRAS, 180, 567

Subrahmanyan, R., Beasley, A. J., Goss, W. M., Golap, K., \& Hunstead, R. W. 2003, AJ, 125, 1095, arXiv:astro-ph/0212154

Subrahmanyan, R., Saripalli, L., \& Hunstead, R. W. 1996, MNRAS, 279, 257

Swarup, G. 1984, JA\&A, 5, 139

Tadhunter et al. 1988, MNRAS, 235, 403

Tateyama, C. E. \& Strauss, F. M. 1992, MNRAS, 256, 8

Taylor, G. B., Barton, E. J., \& Ge, J. P. 1994, AJ, 107, 1942

Taylor, G. B., Fabian, A. C., \& Allen, S. W. 2002, MNRAS, 334, 769, arXiv:astro-ph/0109337

Thompson et al. 1995, ApJS, 101, 259

Tingay et al. 1998, ApJ, 497, 594 
Turtle, A. J. \& Amy, S. W. 1991, in The Magellanic Clouds, ed. R. Haynes \& D. Milne, IAU Symp. 148 (Dordrecht: Kluwer), 114

Ulvestad, J., Johnston, K., Perley, R., \& Fomalont, E. 1981, AJ, 86, 1010

Unewisse, A. M. 1993, PhD thesis, University of Sydney

Van Gorkom, J. H., van der Hulst, J. M., Haschick, A. D., \& Tubbs, A. D. 1990, AJ, 99, 1781

Villar-Martín, M., Tadhunter, C., Morganti, R., Clark, N., Killeen, N., \& Axon, D. 1998, A\&A, 332, 479

Wall, J. V. \& Cole, D. J. 1973, Aust. J. Phys., 26, 881

Wall, J. V. \& Schilizzi, R. T. 1979, MNRAS, 189, 593

Wall, J. V., Shimmins, A. J., \& Bolton, J. G. 1975, Aust. J. Phys. Astrophys. Suppl., 34, 55

Wardle, J. F., Moore, R. L., \& Angel, J. R. P. 1984, ApJ, 279, 93

White, G. L., McAdam, W. B., \& Jones, I. G. 1984, Proc. Astron. Soc. Aust., 5, 507

Whiteoak, J. B. \& Bunton, J. D. 1985, Proc. Astron. Soc. Aust., 6, 171

Whiteoak, J. B. \& Gardner, F. F. 1968, ApJ, 154, 807

Whiteoak, J. B. \& Wilson, W. E. 1990, MNRAS, 245, 665

Wills, B. J. 1973, ApJ, 180, 335

—. 1975, Aust. J. Phys. Astrophys. Suppl., 38, 1

Wright, A. E., Griffith, M. R., Burke, B. F., \& Ekers, R. D. 1994, ApJS, 91, 111

Wright, A. E., Griffith, M. R., Hunt, A. J., Troup, E., Burke, B. F., \& Ekers, R. D. 1996, ApJS, 103, 145

Wright, A. E., Savage, A., \& Bolton, J. G. 1977, Aust. J. Phys. Astrophys. Suppl., 41, 1

Wyllie, D. V. 1969a, MNRAS, 142, 229

—. 1969b, Proc. Astron. Soc. Aust., 1, 234 


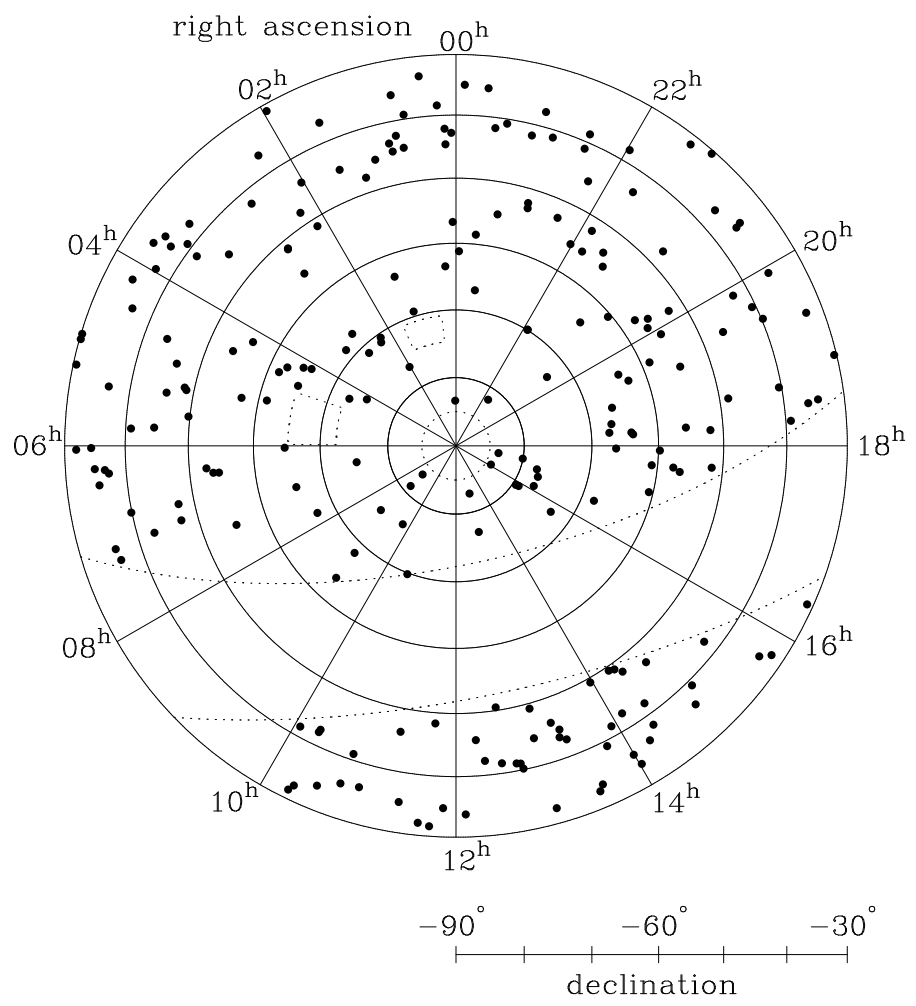

Fig. 1.- Plot of the source locations on a Lambert equal-area projection of the sky. The South Celestial Pole is at the centre of the plot. Dotted lines mark the borders of regions excluded from the sample: the Galactic plane, the circumpolar region, and the Magellanic Clouds. 


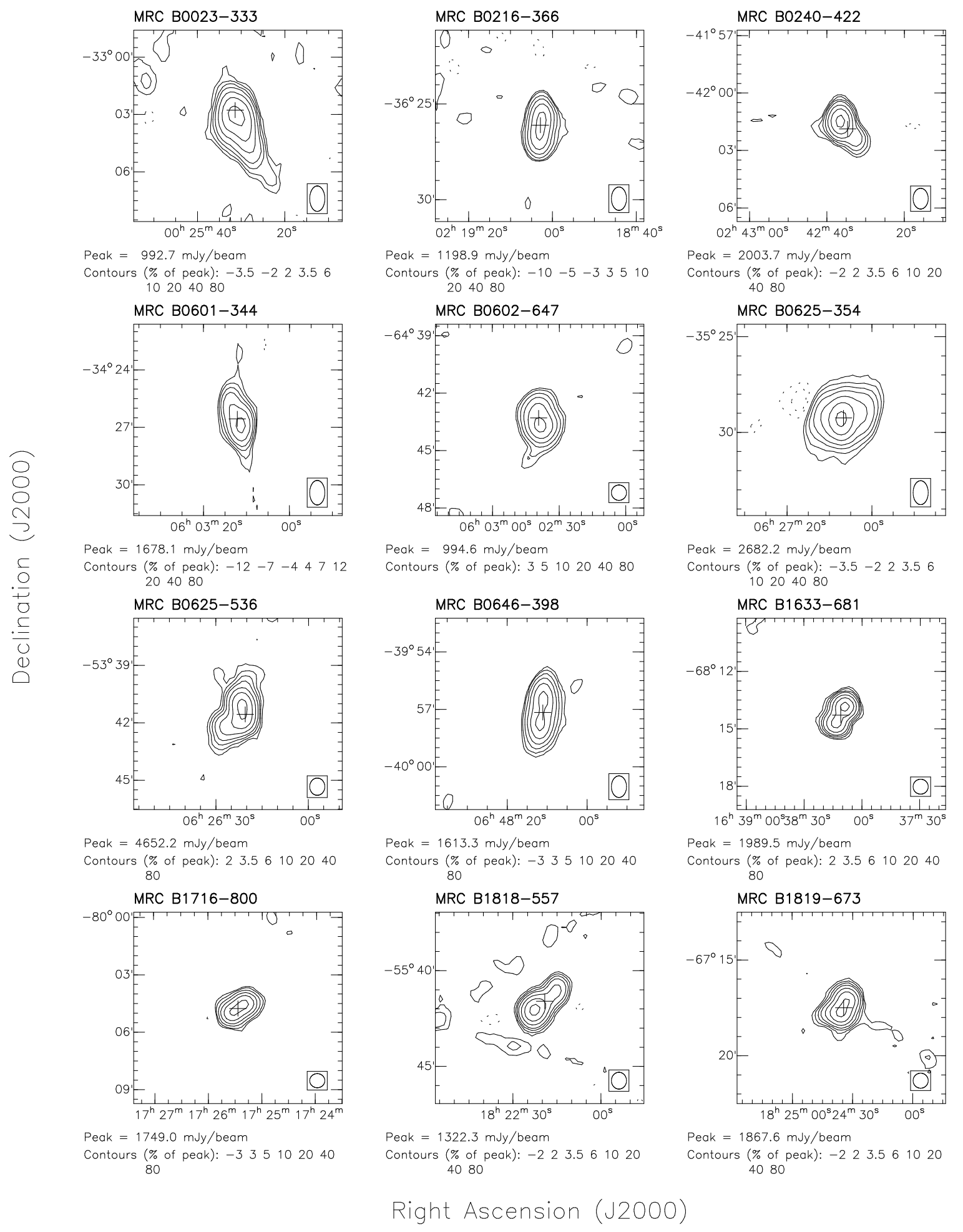

Fig. 2.- $843 \mathrm{MHz}$ contour images of slightly extended MS4 sources, obtained in CUTS observing mode (see $\S 3.1$ ). The crosses mark the positions of the optical counterparts. The Gaussian restoring beam is shown in the bottom right-hand corner of each plot. The side length of each plot is $10^{\prime}$. 


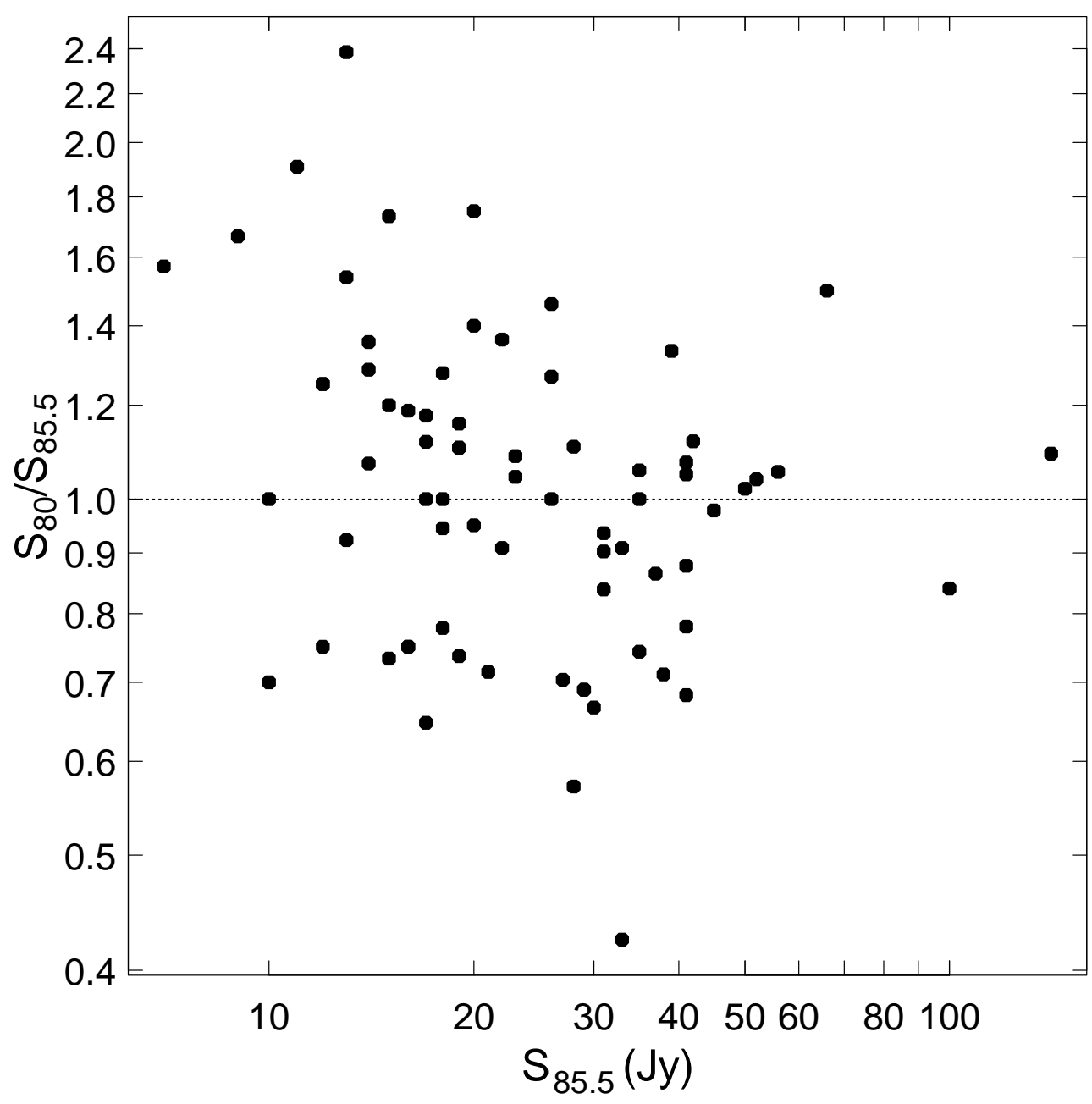

Fig. 3.- Ratio of $S_{80 \mathrm{MHz}}$ to $S_{85.5 \mathrm{MHz}}$ versus $S_{85.5 \mathrm{MHz}}$. The values at $80 \mathrm{MHz}$ were measured with the Culgoora Circular Array, and those at $85.5 \mathrm{MHz}$ were from the MSH survey. 

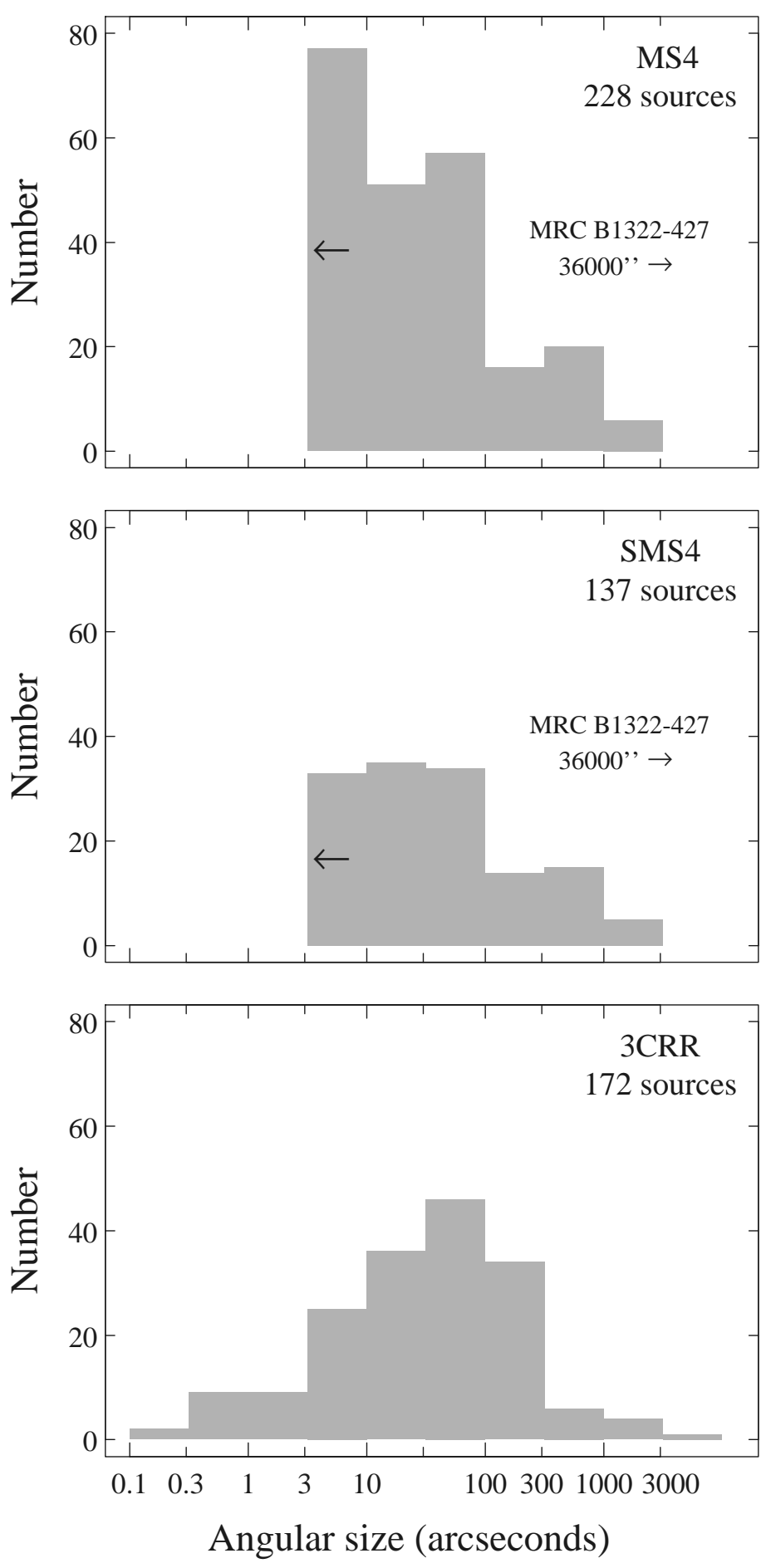

Fig. 4.- Histograms of angular size for the MS4, SMS4, and 3CRR samples. In the MS4 and SMS4 data, MRC B1322-427 is off-scale, and the leftmost bin represents an upper limit to the angular size for many of the sources. 

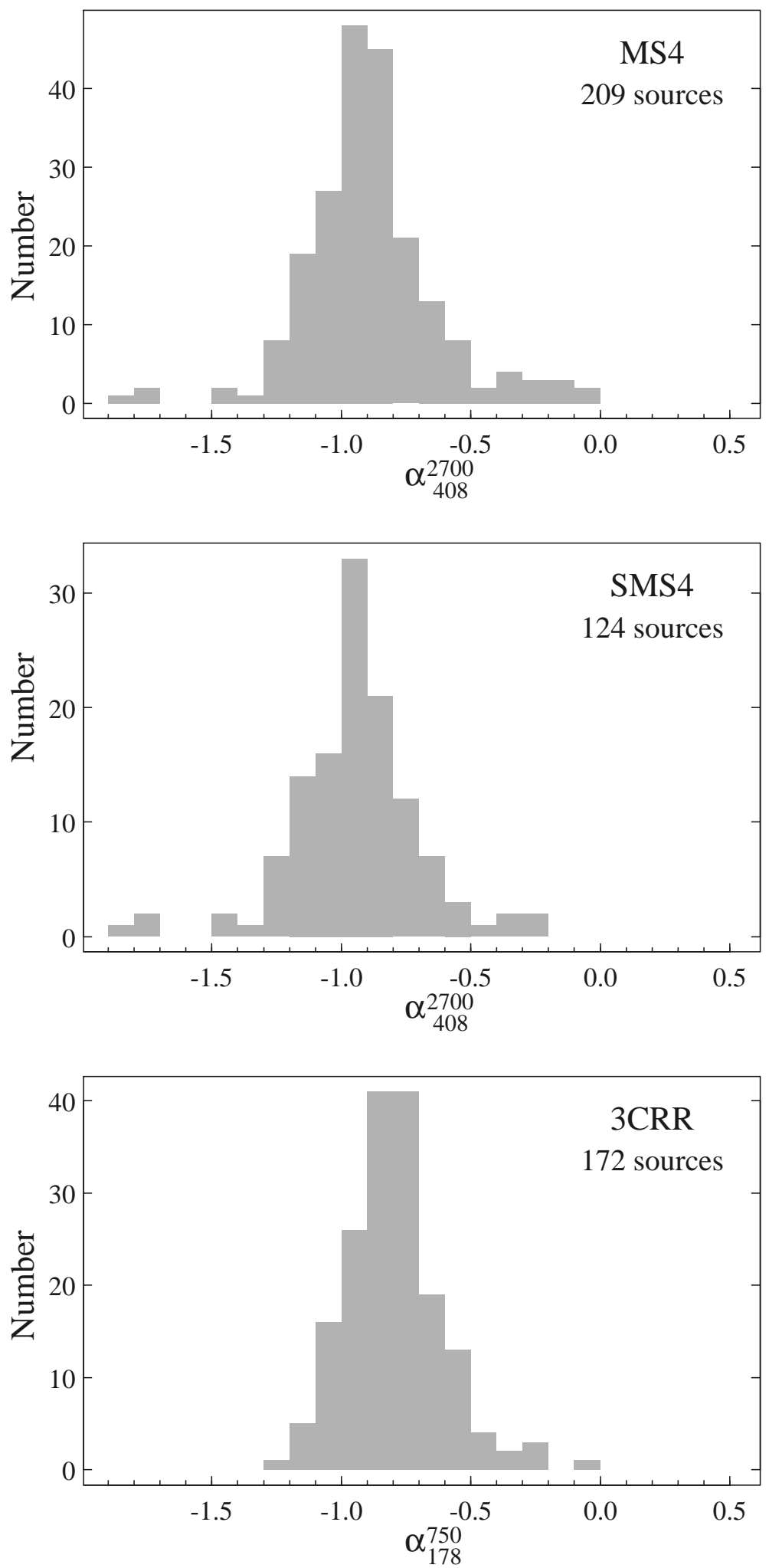

Fig. 5.- Histograms of spectral index for the MS4, SMS4, and 3CRR samples. MS4 and SMS4 sources without reliable flux density data have been omitted. 
Table 1. Radio surveys of strong southern extragalactic sources

\begin{tabular}{ccccl}
\hline \hline $\begin{array}{c}(1) \\
\text { Survey }\end{array}$ & $\begin{array}{c}(2) \\
\text { Frequency } \\
(\mathrm{MHz})\end{array}$ & $\begin{array}{c}(3) \\
\text { Flux density } \\
\text { limit (Jy) }\end{array}$ & $\begin{array}{c}(4) \\
\text { No. of } \\
\text { sources }\end{array}$ & $\begin{array}{c}(5) \\
\text { References }\end{array}$ \\
\hline MSH & 85.5 & 10 & 2270 & 123 \\
PKS & $408^{\mathrm{a}}$ & $4^{\mathrm{b}}$ & 2133 & 45678 \\
PKS 2700 & 2700 & $\sim 0.25^{\mathrm{c}}$ & $\sim 6000^{\mathrm{d}}$ & 9 \\
MRC & 408 & $1.0^{\mathrm{e}}$ & 12141 & 10 \\
PMN & 4850 & $0.04^{\mathrm{f}}$ & 50814 & 11121314 \\
\hline
\end{tabular}

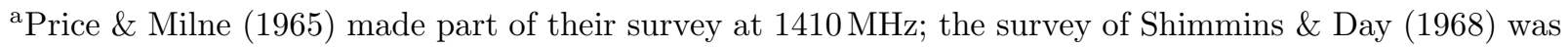
made at $635 \mathrm{MHz}$.

${ }^{\mathrm{b}}$ The flux-density limit at $408 \mathrm{MHz}$ varied from 3 to $4 \mathrm{Jy}$; some papers contained additional sources down to $S_{408}=1.0 \mathrm{Jy}$.

${ }^{\mathrm{c}}$ The flux-density limit varies from 0.1 to $0.6 \mathrm{Jy}$.

${ }^{\mathrm{d}}$ The total number of entries in parts 1 to 14 of the catalogue is 6842 , but some sources were observed more than once.

${ }^{\mathrm{e}}$ Complete to $1 \mathrm{Jy}$ but contains sources down to $0.7 \mathrm{Jy}$.

${ }^{\mathrm{f}}$ The flux-density limit varies from 0.020 to $0.072 \mathrm{Jy}$ with declination.

References. - (1) Mills et al. (1958), (2) Mills et al. (1960), (3) Mills et al. (1961), (4) Bolton et al. (1964), (5) Price \& Milne (1965), (6) Day et al. (1966), (7) Shimmins et al. (1966), (8) Shimmins \& Day (1968), (9) Bolton et al. (1979) and references therein, (10) Large et al. (1981), (11) Griffith et al. (1994), (12) Wright et al. (1994), (13) Griffith et al. (1995), (14) Wright et al. (1996). 
Table 2. Extended sources with $\mathrm{MRC} S_{408}<4.0 \mathrm{Jy}$ which have been added to the sample

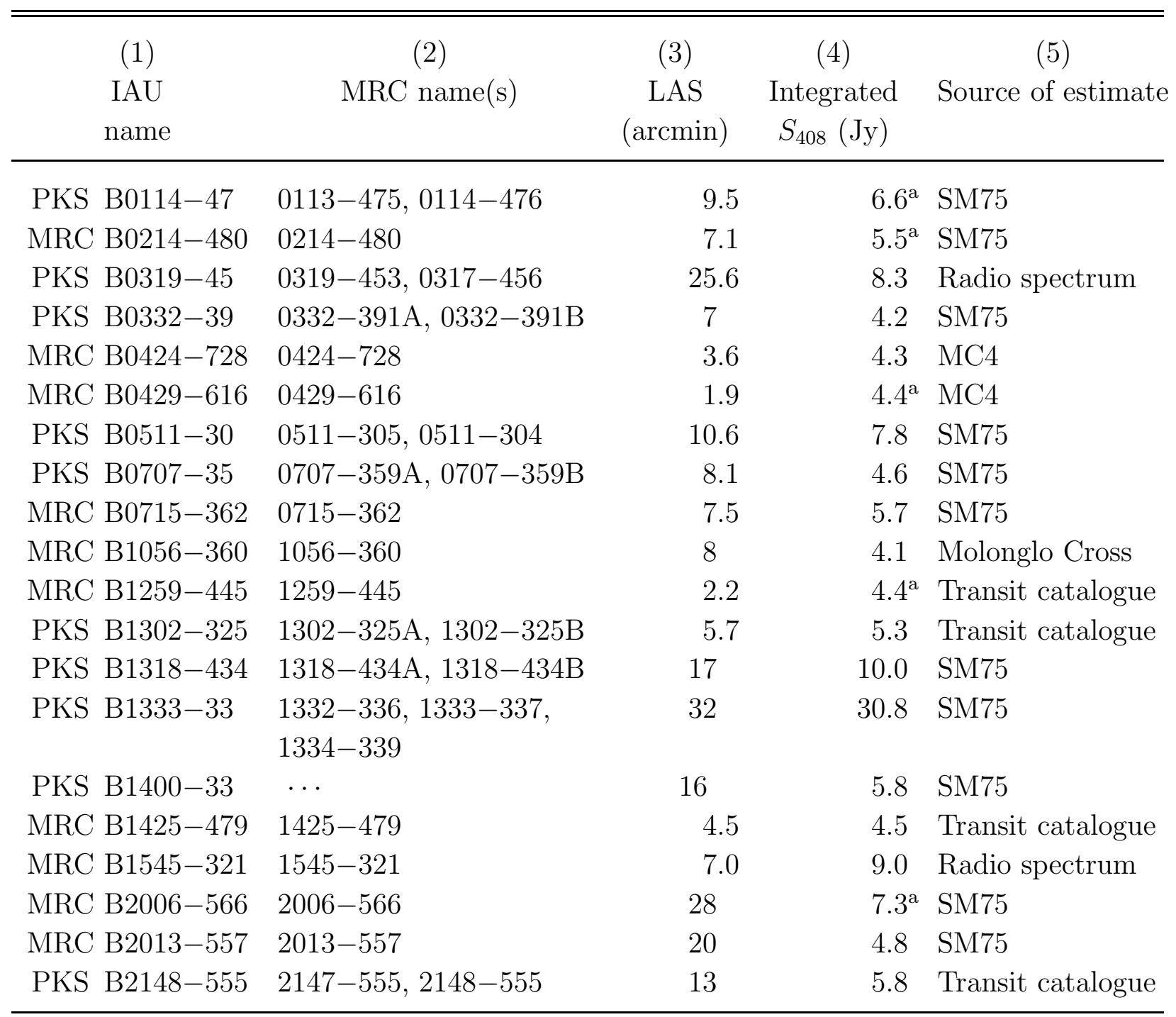

${ }^{\text {aPKS }} 408 \mathrm{MHz}$ flux density was preferred. 
Table 3. Extended sources found to be just below the 4.0 Jy flux-density limit

\begin{tabular}{crrl}
\hline \hline $\begin{array}{c}(1) \\
\text { Source }\end{array}$ & $\begin{array}{c}(2) \\
\text { LAS } \\
\text { name }\end{array}$ & $\begin{array}{c}(3) \\
S_{408}\end{array}$ & \multicolumn{1}{c}{$\begin{array}{c}(4) \\
\text { Means of } \\
\text { estimate }\end{array}$} \\
\hline MRC B0816-705 & 6 & 3.5 & MC4 \\
MRC B1011-316 & 1.3 & 3.9 & Transit catalogue \\
MRC B1148-353 & 1.8 & 3.8 & Transit catalogue \\
MRC B1308-441 & 14.4 & $\sim 3.8$ & Radio spectrum \\
MRC B1511-315 & 2.1 & 3.8 & Radio spectrum \\
MRC B1821-583 & 3.3 & 3.3 & SM75 \\
\hline
\end{tabular}


Table 4. List of sources in the MS4 sample.

\begin{tabular}{|c|c|c|c|c|}
\hline $\begin{array}{c}(1) \\
\text { MRC } \\
\text { name(s) }\end{array}$ & $\begin{array}{c}(2) \\
\text { PKS } \\
\text { name(s) }\end{array}$ & $\begin{array}{c}(3) \\
\text { MSH } \\
\text { name }\end{array}$ & $\begin{array}{l}(4) \\
S_{408} \\
(\mathrm{Jy})\end{array}$ & $\begin{array}{l}\quad(5) \\
\text { References for } \\
\text { radio images }\end{array}$ \\
\hline В0003-567 & В0003-56 & $00-51$ & $5.19 \pm 0.23$ & $\ldots$ \\
\hline B0003-428 & B0003-42 & $00-42$ & $5.15 \pm 0.16$ & $\ldots$ \\
\hline B0003-833 & B0004-83 & $\ldots$ & $6.62 \pm 0.31$ & 1 \\
\hline B0007-446 & B0007-44 & $00-43$ & $6.81 \pm 0.21$ & $\ldots$ \\
\hline B0008-421 & B0008-42 & $\ldots$ & $6.61 \pm 0.21$ & $\ldots$ \\
\hline B0012-383 & B0012-38 & $00-35$ & $4.87 \pm 0.14$ & $\ldots$ \\
\hline B0013-634 & B0013-63 & $00-61$ & $6.90 \pm 0.17$ & $\ldots$ \\
\hline B0023-333 & B0023-33 & $00-38$ & $4.09 \pm 0.18$ & 2 \\
\hline B0036-392 & B0035-39 & $00-313$ & $5.80 \pm 0.15$ & $\ldots$ \\
\hline B0039-445 & B0039-44 & $00-410$ & $10.23 \pm 0.31$ & 3 \\
\hline B0042-357 & B0042-35 & $00-315$ & $5.79 \pm 0.18$ & $\ldots$ \\
\hline B0043-424 & B0043-42 & $00-411$ & $21.0 \pm 1.2$ & 1345 \\
\hline B0048-447 & B0048-44 & $00-413$ & $4.78 \pm 0.15$ & $\ldots$ \\
\hline B0049-433 & B0049-43 & $00-414$ & $8.07 \pm 0.25$ & $\ldots$ \\
\hline B0103-453 & B0103-45 & $01-41$ & $7.5 \pm 0.7$ & 14 \\
\hline B0110-692 & B0110-69 & $\ldots$ & $6.10 \pm 0.19$ & 1 \\
\hline $\left.\begin{array}{l}\text { B0113-475 } \\
\text { B0114-476 }\end{array}\right\}$ & B0114-47 & $01-45$ & $10.4 \pm 0.8$ & 16789 \\
\hline B0119-634 & B0119-63 & $01-61$ & $5.20 \pm 0.23$ & $\cdots$ \\
\hline B0131-449 & B0131-44 & $01-49$ & $4.03 \pm 0.13$ & $\ldots$ \\
\hline $\left.\begin{array}{l}\mathrm{B} 0131-367 \mathrm{~A} \\
\mathrm{~B} 0131-367 \mathrm{~B}\end{array}\right\}$ & B0131-36 & $01-311$ & $17.1 \pm 1.0$ & 12461011 \\
\hline & & & & 12 \\
\hline B0157-311 & B0157-31 & $01-315$ & $10.31 \pm 0.45$ & 12 \\
\hline B0201-440 & B0201-44 & $02-41$ & $6.17 \pm 0.27$ & $\ldots$ \\
\hline B0202-765 & B0202-76 & $\cdots$ & $8.46 \pm 0.08$ & $\ldots$ \\
\hline B0208-512 & B0208-512 & $\ldots$ & $5.48 \pm 0.14$ & 13 \\
\hline B0214-480 & B0214-48 & $02-43$ & $9.5 \pm 0.8$ & 161415 \\
\hline B0216-366 & B0216-36 & $02-33$ & $4.3 \pm 0.3$ & 6 \\
\hline B0219-706 & B0219-706 & $\ldots$ & $6.87 \pm 0.29$ & $\ldots$ \\
\hline B0223-712 & B0223-71 & $\ldots$ & $5.28 \pm 0.23$ & $\ldots$ \\
\hline B0240-422 & B0240-42 & $02-410$ & $4.22 \pm 0.13$ & $\ldots$ \\
\hline B0241-512 & B0241-513 & $\ldots$ & $4.45 \pm 0.12$ & 6 \\
\hline B0242-514 & B0241-51 & $02-53$ & $7.99 \pm 0.24$ & 6 \\
\hline В0245-558 & B $0245-55$ & $02-54$ & $8.25 \pm 0.36$ & $\ldots$ \\
\hline B0251-675 & B0251-67 & $02-65$ & $4.85 \pm 0.15$ & $\ldots$ \\
\hline B0252-712 & B0252-71 & $02-72$ & $14.08 \pm 0.43$ & $\ldots$ \\
\hline B0315-685 & B0315-68 & $\ldots$ & $5.26 \pm 0.14$ & $\ldots$ \\
\hline $\left.\begin{array}{l}\text { B0317-456 } \\
\text { B0319-453 }\end{array}\right\}$ & B0319-45 & $03-43$ & $8.3 \pm 2.0$ & 14161718 \\
\hline B0320-373 & B0320-37 & $03-31$ & $259 \pm 26$ & $\begin{array}{l}1219202122 \\
23242526\end{array}$ \\
\hline
\end{tabular}


Table 4-Continued

\begin{tabular}{|c|c|c|c|c|}
\hline $\begin{array}{c}(1) \\
\text { MRC } \\
\text { name(s) }\end{array}$ & $\begin{array}{c}(2) \\
\text { PKS } \\
\text { name(s) }\end{array}$ & $\begin{array}{c}(3) \\
\text { MSH } \\
\text { name }\end{array}$ & $\begin{array}{c}(4) \\
S_{408} \\
(\mathrm{Jy})\end{array}$ & $\begin{array}{l}\quad(5) \\
\text { References for } \\
\text { radio images }\end{array}$ \\
\hline B0332-391B & B0332-39 & $03-32$ & $4.2 \pm 0.3$ & 1226102728 \\
\hline B0335-415 & B0335-415 & $03-44$ & $4.10 \pm 0.50$ & $\ldots$ \\
\hline B0336-355 & B0336-35 & $03-33$ & $5.0 \pm 0.3$ & $\begin{array}{l}12462327 \\
29303132\end{array}$ \\
\hline B0340-372 & B0340-37 & $\ldots$ & $5.03 \pm 0.16$ & $\ldots$ \\
\hline B0344-345 & B0344-34 & $03-36$ & $9.3 \pm 0.8$ & 12461027 \\
\hline B0357-371 & B0357-37 & $03-39$ & $4.68 \pm 0.15$ & 1 \\
\hline B0407-658 & B0408-65 & $04-62$ & $51.14 \pm 1.72$ & $\ldots$ \\
\hline В0409-752 & B0410-75 & $04-71$ & $35.97 \pm 0.63$ & 3533 \\
\hline B0411-346A & B0411-34 & $04-33$ & $4.35 \pm 0.20$ & $\ldots$ \\
\hline B0411-647 & B0411-647 & $\cdots$ & $4.18 \pm 0.11$ & $\ldots$ \\
\hline B0411-561 & B0411-56 & $04-52$ & $6.79 \pm 0.30$ & 5 \\
\hline B0420-625 & B0420-62 & $04-63$ & $11.54 \pm 0.18$ & 5 \\
\hline B0424-728 & B0425-72 & $04-72$ & $4.3 \pm 0.35$ & 181734 \\
\hline $\left.\begin{array}{l}\text { B0427-539A } \\
\text { B0427-539B }\end{array}\right\}$ & B0427-53 & $04-54$ & $14.6 \pm 0.9$ & 1610121422 \\
\hline & & & & 35 \\
\hline B0427-366 & B $0427-36$ & $04-36$ & $8.41 \pm 1.05$ & $\ldots$ \\
\hline B0429-616 & B0429-61 & $04-64$ & $6.5 \pm 1.2$ & 1173436 \\
\hline B0436-650 & B0437-65 & $04-65$ & $4.26 \pm 0.14$ & 34 \\
\hline B0438-436 & B $0438-43$ & $04-49$ & $8.12 \pm 0.25$ & 37 \\
\hline B0453-301 & B0453-30 & $\cdots$ & $9.16 \pm 0.20$ & $\ldots$ \\
\hline B0454-463 & B0454-46 & $\cdots$ & $4.25 \pm 0.13$ & $\ldots$ \\
\hline B0456-301 & B0456-30 & $\cdots$ & $7.21 \pm 0.36$ & 14 \\
\hline B0506-612 & B0506-61 & $05-61$ & $5.03 \pm 0.16$ & $\ldots$ \\
\hline B0509-573 & B0509-573 & $\ldots$ & $5.12 \pm 0.22$ & $\ldots$ \\
\hline B0511-484 & B0511-48 & $\cdots$ & $6.84 \pm 0.11$ & 161038 \\
\hline $\left.\begin{array}{l}\text { B0511-304 } \\
\text { B0511-305 }\end{array}\right\}$ & B0511-30 & $05-35$ & $7.8 \pm 0.5$ & 1246827 \\
\hline & & & & 3940 \\
\hline B0513-488 & B0513-488 & $\cdots$ & $4.04 \pm 0.11$ & $\ldots$ \\
\hline $\left.\begin{array}{l}\mathrm{B} 0518-458 \mathrm{~A} \\
\mathrm{~B} 0518-458 \mathrm{~B}\end{array}\right\}$ & B0518-45 & $05-43$ & $166 \pm 8.3$ & 146142241 \\
\hline B0521-365 & B0521-36 & $05-36$ & $36.08 \pm 1.56$ & $\begin{array}{l}42434445 \\
24647484950\end{array}$ \\
\hline B0534-497 & B0535-49 & $05-46$ & $6.69 \pm 0.15$ & $\ldots$ \\
\hline B0546-445 & B0546-44 & $05-48$ & $4.41 \pm 0.14$ & $\ldots$ \\
\hline B0547-408 & B $0547-40$ & $05-410$ & $8.24 \pm 0.24$ & $\ldots$ \\
\hline B0601-344 & B0600-34 & $06-31$ & $4.40 \pm 0.46$ & $\ldots$ \\
\hline B0602-319 & B0602-31 & $\cdots$ & $6.67 \pm 0.29$ & 32 \\
\hline B0602-647 & B0602-64 & $\ldots$ & $4.49 \pm 0.10$ & $\ldots$ \\
\hline B0614-349 & B0614-34 & $06-36$ & $5.29 \pm 0.21$ & $\ldots$ \\
\hline B0615-365 & B0615-365 & $\ldots$ & $4.41 \pm 0.20$ & $\ldots$ \\
\hline B0618-371 & B0618-37 & $06-37$ & $5.81 \pm 0.22$ & 1244151 \\
\hline
\end{tabular}


Table 4-Continued

\begin{tabular}{|c|c|c|c|c|}
\hline $\begin{array}{c}(1) \\
\text { MRC } \\
\text { name(s) }\end{array}$ & $\begin{array}{c}(2) \\
\text { PKS } \\
\text { name(s) }\end{array}$ & $\begin{array}{c}(3) \\
\mathrm{MSH} \\
\text { name }\end{array}$ & $\begin{array}{c}(4) \\
S_{408} \\
(\mathrm{Jy})\end{array}$ & $\begin{array}{l}(5) \\
\text { References for } \\
\text { radio images }\end{array}$ \\
\hline B0620-526 & B $0620-52$ & $06-53$ & $9.3 \pm 0.8$ & 11217 \\
\hline B0625-536 & B0625-53 & $06-55$ & $26 \pm 1.4$ & 35525354 \\
\hline B0625-354 & B0625-35 & $06-38$ & $9.23 \pm 0.70$ & 242728 \\
\hline B0625-545 & B0625-545 & $\ldots$ & $7.86 \pm 0.68$ & 1 \\
\hline B0637-752 & B0637-75 & $06-71$ & $7.89 \pm 0.10$ & 375556 \\
\hline B0646-398 & B0646-39 & $06-312$ & $7.0 \pm 0.7$ & $\ldots$ \\
\hline B0647-475 & B0647-475 & $\ldots$ & $4.35 \pm 0.11$ & $\ldots$ \\
\hline B0658-656 & B0658-65 & $06-64$ & $6.14 \pm 0.24$ & $\ldots$ \\
\hline B0700-473 & B0700-47 & $07-41$ & $4.33 \pm 0.11$ & $\ldots$ \\
\hline B0704-427 & B0704-42 & $07-43$ & $4.47 \pm 0.12$ & $\ldots$ \\
\hline $\left.\begin{array}{l}\mathrm{B} 0707-359 \mathrm{~A} \\
\mathrm{~B} 0707-359 \mathrm{~B}\end{array}\right\}$ & B $0707-35$ & $07-34$ & $4.6 \pm 0.3$ & 1681027 \\
\hline B0715-362 & B0715-36 & $07-35$ & $5.7 \pm 0.3$ & 14627 \\
\hline B0719-553 & B0719-55 & $07-53$ & $5.63 \pm 0.18$ & 1 \\
\hline B0743-673 & B0744-67 & $\ldots$ & $8.61 \pm 1.04$ & 557 \\
\hline B0842-754 & B0842-75 & $08-71$ & $13.35 \pm 0.58$ & $\ldots$ \\
\hline B0842-835 & B0841-83 & $\ldots$ & $4.78 \pm 0.21$ & $\ldots$ \\
\hline B0846-811 & B0846-81 & $\ldots$ & $4.09 \pm 0.18$ & $\ldots$ \\
\hline B0906-682 & B0905-68 & $\cdots$ & $6.11 \pm 0.07$ & $\ldots$ \\
\hline B0910-636 & B0910-63 & $\ldots$ & $4.01 \pm 0.10$ & $\ldots$ \\
\hline B0943-761 & B0943-76 & $\ldots$ & $4.91 \pm 0.22$ & $\ldots$ \\
\hline B1003-415 & B1003-415 & $\ldots$ & $4.07 \pm 0.18$ & $\ldots$ \\
\hline B1015-314 & B1015-31 & $\ldots$ & $9.67 \pm 0.35$ & $\ldots$ \\
\hline B1017-421 & B1017-42 & $\ldots$ & $5.04 \pm 0.22$ & 4 \\
\hline B1017-325 & B1017-325 & $10-36$ & $4.76 \pm 0.13$ & $\ldots$ \\
\hline B1017-426 & B1018-42 & $\ldots$ & $12.72 \pm 0.55$ & 58 \\
\hline B1030-340 & B1030-34 & $10-38$ & $5.59 \pm 0.25$ & $\ldots$ \\
\hline B1036-697 & B1036-69 & $\ldots$ & $6.92 \pm 0.27$ & $\ldots$ \\
\hline B1044-357 & B1044-357 & $\ldots$ & $4.15 \pm 0.13$ & $\ldots$ \\
\hline B1046-409 & B1046-409 & $\ldots$ & $5.02 \pm 0.16$ & 13 \\
\hline B1056-360 & B1056-360 & $10-314$ & $4.09 \pm 0.15$ & 12417 \\
\hline B1116-462 & B1116-46 & $\ldots$ & $4.68 \pm 0.21$ & $\ldots$ \\
\hline B1123-351 & B1123-35 & $11-33$ & $6.61 \pm 0.28$ & 124 \\
\hline B1136-320 & B1136-32 & $11-38$ & $6.78 \pm 0.30$ & 15 \\
\hline B1143-483 & B1143-48 & $11-46$ & $7.47 \pm 0.23$ & $\ldots$ \\
\hline B1143-316 & B1143-31 & $11-310$ & $5.77 \pm 0.25$ & 146 \\
\hline B1151-348 & B1151-34 & $11-314$ & $10.90 \pm 0.40$ & 32 \\
\hline B1206-337 & B1206-337 & $12-32$ & $4.65 \pm 0.21$ & $\ldots$ \\
\hline B1215-457 & B1215-45 & $\ldots$ & $9.59 \pm 0.42$ & $\ldots$ \\
\hline B1221-423 & B1221-42 & $\ldots$ & $5.08 \pm 0.13$ & 559 \\
\hline B1232-416 & B1232-41 & $\ldots$ & $5.33 \pm 0.24$ & $\ldots$ \\
\hline B1234-504 & $\ldots$ & $\ldots$ & $4.57 \pm 0.08$ & $\ldots$ \\
\hline B1243-412 & B1243-412 & $\ldots$ & $4.40 \pm 0.14$ & $\ldots$ \\
\hline B1246-410 & B1245-41 & $12-45$ & $10.59 \pm 0.46$ & 606162 \\
\hline
\end{tabular}


Table 4-Continued

\begin{tabular}{|c|c|c|c|c|}
\hline $\begin{array}{c}(1) \\
\text { MRC } \\
\text { name(s) }\end{array}$ & $\begin{array}{c}(2) \\
\text { PKS } \\
\text { name(s) }\end{array}$ & $\begin{array}{c}(3) \\
\text { MSH } \\
\text { name }\end{array}$ & $\begin{array}{l}(4) \\
S_{408} \\
(\mathrm{Jy})\end{array}$ & $\begin{array}{l}\quad(5) \\
\text { References for } \\
\text { radio images }\end{array}$ \\
\hline B1247-401 & B1247-40 & $\cdots$ & $6.03 \pm 0.18$ & $\ldots$ \\
\hline B1259-769 & B1259-76 & $\ldots$ & $4.15 \pm 0.13$ & $\ldots$ \\
\hline B1259-445 & B1259-44 & $\cdots$ & $4.2 \pm 0.6$ & 1 \\
\hline $\left.\begin{array}{l}\mathrm{B} 1302-325 \mathrm{~A} \\
\mathrm{~B} 1302-325 \mathrm{~B}\end{array}\right\}$ & B1302-325 & $\cdots$ & $5.27 \pm 1.42$ & 117 \\
\hline B1302-491 & B1302-49 & $13-41$ & $12.6 \pm 1.26$ & $\begin{array}{l}12363646566 \\
67686970 \quad 7172\end{array}$ \\
\hline B1303-827 & B1302-82 & $\cdots$ & $4.84 \pm 0.22$ & $\ldots$ \\
\hline B1315-460 & $\ldots$ & $\cdots$ & $6.24 \pm 0.27$ & $\ldots$ \\
\hline $\left.\begin{array}{l}\mathrm{B} 1318-434 \mathrm{~A} \\
\mathrm{~B} 1318-434 \mathrm{~B}\end{array}\right\}$ & B1318-434 & $\cdots$ & $10.0 \pm 0.6$ & $\begin{array}{l}1610127374 \\
7576\end{array}$ \\
\hline B1320-446 & B1320-446 & $\cdots$ & $6.91 \pm 0.55$ & $\ldots$ \\
\hline B1322-427 & B1322-42 & $13-42$ & $2740 \pm 548$ & $\begin{array}{lllllll}1 & 4 & 13 & 14 & 19 & 20 \\
21 & 22 & 23 & 39 & 41 & 42 \\
77 & 78 & 79 & 80 & 81 & 82 \\
83 & 84 & 85 & 86 & 87 & 88 \\
89 & 90 & 91 & 92 & 93 & 94 \\
95 & 96 & 97 & 98 & 99 & 100\end{array}$ \\
\hline B1330-328 & B1330-328 & $\cdots$ & $4.27 \pm 0.14$ & 36 \\
\hline $\left.\begin{array}{l}\mathrm{B} 1332-336 \\
\mathrm{~B} 1333-337 \\
\mathrm{~B} 1334-339\end{array}\right\}$ & $\left.\begin{array}{l}\mathrm{B} 1332-33 \\
\mathrm{~B} 1333-33 \\
\mathrm{~B} 1334-33\end{array}\right\}$ & $13-33$ & $30.8 \pm 1.8$ & $\begin{array}{l}\begin{array}{lll}124612 & 22 \\
40 & 101 \\
40 & 101\end{array}\end{array}$ \\
\hline B1346-391 & B1346-39 & $13-44$ & $5.84 \pm 0.26$ & $\ldots$ \\
\hline B1355-416 & B1355-41 & $13-45$ & $12.9 \pm 0.6$ & 15 \\
\hline B1358-493 & $\ldots$ & $\ldots$ & $6.46 \pm 0.28$ & $\ldots$ \\
\hline B1359-358 & B1359-35 & $\ldots$ & $4.47 \pm 0.14$ & $\ldots$ \\
\hline$\cdots$ & B1400-33 & $14-32$ & $5.8 \pm 0.3$ & $\begin{array}{l}261027102103 \\
104\end{array}$ \\
\hline B1407-425 & B1407-425 & $\cdots$ & $4.67 \pm 0.21$ & $\ldots$ \\
\hline B1413-364 & B1413-36 & $\cdots$ & $5.71 \pm 0.22$ & 124 \\
\hline B1416-493 & B1416-49 & $\ldots$ & $7.34 \pm 0.32$ & $\ldots$ \\
\hline B1421-382 & B1421-38 & $14-34$ & $6.92 \pm 0.21$ & 1 \\
\hline B1421-490 & $\ldots$ & $\ldots$ & $13.10 \pm 0.67$ & $\ldots$ \\
\hline B1424-418 & B1424-41 & $\cdots$ & $6.39 \pm 0.28$ & 13 \\
\hline B1425-479 & $\ldots$ & $14-46$ & $4.45 \pm 0.37$ & 117 \\
\hline B1445-468 & B1445-46 & $14-49$ & $6.97 \pm 0.31$ & $\ldots$ \\
\hline B1451-364 & B1451-36 & $14-38$ & $8.99 \pm 0.72$ & 145 \\
\hline B1458-391 & $\ldots$ & $\cdots$ & $6.07 \pm 0.19$ & $\ldots$ \\
\hline B1526-423 & $\ldots$ & $15-43$ & $17.86 \pm 1.38$ & $\ldots$ \\
\hline B1540-730 & B1540-73 & $\ldots$ & $5.33 \pm 0.14$ & $\ldots$ \\
\hline B1540-337 & B1540-337 & $\cdots$ & $4.33 \pm 0.19$ & $\ldots$ \\
\hline B1545-321 & B1545-321 & $\cdots$ & $9.0 \pm 2.2$ & 14817105 \\
\hline
\end{tabular}


Table 4-Continued

\begin{tabular}{|c|c|c|c|c|}
\hline $\begin{array}{c}(1) \\
\text { MRC } \\
\text { name(s) }\end{array}$ & $\begin{array}{c}(2) \\
\text { PKS } \\
\text { name(s) }\end{array}$ & $\begin{array}{c}(3) \\
\text { MSH } \\
\text { name }\end{array}$ & $\begin{array}{l}(4) \\
S_{408} \\
(\mathrm{Jy})\end{array}$ & $\begin{array}{c}\quad(5) \\
\text { References for } \\
\text { radio images }\end{array}$ \\
\hline B1547-795 & B1547-79 & $\cdots$ & $10.45 \pm 0.57$ & 5 \\
\hline B1549-790 & B1549-79 & $\cdots$ & $7.95 \pm 0.35$ & $\ldots$ \\
\hline B1607-841 & B1606-84 & $\cdots$ & $4.91 \pm 0.22$ & $\ldots$ \\
\hline B1610-771 & B1610-77 & $\ldots$ & $5.35 \pm 0.17$ & $\ldots$ \\
\hline B1622-310 & B1622-31 & $\cdots$ & $5.20 \pm 0.23$ & 32 \\
\hline B1633-681 & B1632-68 & $\cdots$ & $6.66 \pm 0.29$ & $\ldots$ \\
\hline B1637-771 & B1637-77 & $\cdots$ & $13.5 \pm 1.4$ & 11217 \\
\hline B1655-776 & B1655-77 & $\cdots$ & $5.04 \pm 0.22$ & 13 \\
\hline B1706-606 & $\cdots$ & $\cdots$ & $5.54 \pm 0.17$ & $\ldots$ \\
\hline B1716-800 & B1716-80 & $\ldots$ & $5.91 \pm 0.26$ & $\ldots$ \\
\hline B1721-836 & B1720-83 & $\cdots$ & $4.63 \pm 0.21$ & $\ldots$ \\
\hline $\begin{array}{l}\text { B1733-565A } \\
\text { B1733-565B }\end{array}$ & B1733-56 & $\cdots$ & $20.3 \pm 1.2$ & 16121441106 \\
\hline & & & & 107 \\
\hline B1737-575 & B1737-575 & $\cdots$ & $5.12 \pm 0.64$ & $\ldots$ \\
\hline B1737-609 & B1737-60 & $\cdots$ & $8.32 \pm 0.21$ & 1 \\
\hline B1740-517 & B1740-517 & $\ldots$ & $5.38 \pm 0.12$ & $\ldots$ \\
\hline B1754-597 & B1754-59 & $17-51$ & $12.58 \pm 0.55$ & $\ldots$ \\
\hline B1756-663 & B1756-663 & $\ldots$ & $5.46 \pm 0.22$ & $\ldots$ \\
\hline B1814-519 & B1814-51 & $18-52$ & $14.26 \pm 0.28$ & $\ldots$ \\
\hline B1814-637 & B1814-63 & $\cdots$ & $37.00 \pm 1.34$ & $\ldots$ \\
\hline B1817-391 & $\ldots$ & $18-33$ & $9.74 \pm 0.24$ & 108 \\
\hline B1817-640 & B1817-64 & $\cdots$ & $11.50 \pm 0.35$ & $\ldots$ \\
\hline B1818-557 & B1818-557 & $18-53$ & $4.14 \pm 0.19$ & $\ldots$ \\
\hline B1819-673 & B1819-67 & $\ldots$ & $6.06 \pm 0.19$ & 5 \\
\hline B1827-360 & B1827-36 & $\cdots$ & $25.83 \pm 0.79$ & $\ldots$ \\
\hline B1829-344 & B1829-344 & $\cdots$ & $4.19 \pm 0.19$ & $\ldots$ \\
\hline B1831-668 & B1831-668 & $\ldots$ & $4.28 \pm 0.14$ & $\ldots$ \\
\hline B1839-487 & B1839-48 & $18-43$ & $9.08 \pm 0.23$ & 12 \\
\hline B1840-404 & B1840-40 & $18-44$ & $8.44 \pm 0.24$ & 15 \\
\hline B1853-303 & B1853-303 & $\cdots$ & $5.09 \pm 0.23$ & $\ldots$ \\
\hline B1854-663 & B1855-66 & $\ldots$ & $4.41 \pm 0.14$ & $\ldots$ \\
\hline B1917-546 & B1917-54 & $19-52$ & $4.59 \pm 0.21$ & $\ldots$ \\
\hline B1922-627 & B1922-62 & $19-61$ & $4.88 \pm 0.20$ & 1 \\
\hline B1923-328 & B1923-328 & $\ldots$ & $4.25 \pm 0.18$ & $\ldots$ \\
\hline B1929-397 & B1929-397 & $\cdots$ & $6.41 \pm 0.27$ & 12427 \\
\hline B1932-464 & B1932-46 & $19-46$ & $39.61 \pm 0.83$ & 5109 \\
\hline B1933-587 & B1933-58 & $19-56$ & $9.13 \pm 0.40$ & $\ldots$ \\
\hline B1934-638 & B1934-63 & $\ldots$ & $6.24 \pm 0.20$ & $\ldots$ \\
\hline B1940-406 & B1940-40 & $19-410$ & $5.7 \pm 0.3$ & 146110 \\
\hline B1953-425 & B1953-42 & $19-413$ & $10.13 \pm 0.95$ & $\ldots$ \\
\hline B1954-552 & B1954-55 & $19-57$ & $14.8 \pm 1.0$ & 13514 \\
\hline B1955-357 & B1955-35 & $19-35$ & $4.86 \pm 0.15$ & $\ldots$ \\
\hline B2006-566 & B2006-56 & $20-52$ & $12.2 \pm 0.9$ & 1610111112 \\
\hline
\end{tabular}


Table 4-Continued

\begin{tabular}{|c|c|c|c|c|}
\hline $\begin{array}{c}(1) \\
\text { MRC } \\
\text { name(s) }\end{array}$ & $\begin{array}{c}(2) \\
\text { PKS } \\
\text { name(s) }\end{array}$ & $\begin{array}{c}(3) \\
\text { MSH } \\
\text { name }\end{array}$ & $\begin{array}{l}(4) \\
S_{408} \\
(\mathrm{Jy})\end{array}$ & $\begin{array}{l}\quad(5) \\
\text { References for } \\
\text { radio images }\end{array}$ \\
\hline B2009-524 & B2009-52 & $20-53$ & $4.52 \pm 0.12$ & $\ldots$ \\
\hline B2013-557 & B2014-55 & $20-54$ & $4.8 \pm 0.3$ & 161027 \\
\hline B2020-575 & B2020-57 & $20-55$ & $9.89 \pm 0.39$ & 15 \\
\hline B2028-732 & B2028-73 & $20-71$ & $4.57 \pm 0.15$ & 1 \\
\hline B2031-359 & B2031-359 & $\ldots$ & $4.40 \pm 0.11$ & 2 \\
\hline B2032-350 & B2032-35 & $20-37$ & $17.60 \pm 0.76$ & $\ldots$ \\
\hline B2041-604 & B2041-60 & $20-61$ & $11.16 \pm 0.34$ & 1110 \\
\hline B2049-368 & B2049-36 & $\ldots$ & $5.75 \pm 0.18$ & $\ldots$ \\
\hline B2052-474 & B2052-47 & $\ldots$ & $4.15 \pm 0.10$ & 13 \\
\hline B2059-641 & B2059-64 & $20-62$ & $4.24 \pm 0.17$ & $\ldots$ \\
\hline B2115-305 & B2115-30 & $21-34$ & $6.89 \pm 0.30$ & 32 \\
\hline B2122-555 & $\mathrm{B} 2122-555$ & $21-52$ & $4.05 \pm 0.18$ & $\ldots$ \\
\hline B2128-315 & B2128-315 & $21-36$ & $4.18 \pm 0.13$ & 32 \\
\hline B2140-434 & B2140-43 & $21-47$ & $9.18 \pm 0.28$ & 5 \\
\hline B2140-817 & B2141-81 & $\ldots$ & $8.72 \pm 1.01$ & 5 \\
\hline $\left.\begin{array}{l}\mathrm{B} 2147-555 \\
\mathrm{~B} 2148-555\end{array}\right\}$ & B2148-555 & $\cdots$ & $5.8 \pm 1.0$ & 117113 \\
\hline B2150-520 & B2150-52 & $21-58$ & $10.08 \pm 0.20$ & 5 \\
\hline B2152-699 & B2153-69 & $\ldots$ & $61.6 \pm 3.7$ & $\begin{array}{l}161441114115 \\
116117118\end{array}$ \\
\hline B2153-699 & $\cdots$ & $\cdots$ & $11.9 \pm 2.0$ & $\begin{array}{l}161441114115 \\
116118\end{array}$ \\
\hline B2158-380 & B2158-380 & $21-38$ & $4.12 \pm 0.14$ & 12119 \\
\hline B2201-555 & B2201-55 & $\ldots$ & $5.56 \pm 0.25$ & $\ldots$ \\
\hline B2213-456 & B2213-45 & $\ldots$ & $4.33 \pm 0.10$ & $\ldots$ \\
\hline B2223-528 & B2223-52 & $22-52$ & $8.83 \pm 0.38$ & $\ldots$ \\
\hline B2226-411 & B2226-41 & $\ldots$ & $6.83 \pm 0.79$ & 1 \\
\hline B2226-386 & B2226-38 & $\ldots$ & $8.21 \pm 0.21$ & $\ldots$ \\
\hline B2250-412 & B2250-41 & $22-46$ & $13.93 \pm 0.60$ & 512120 \\
\hline B2252-530 & $\mathrm{B} 2252-53$ & $22-54$ & $6.37 \pm 0.28$ & 1 \\
\hline B2253-522 & B2253-52 & $\ldots$ & $7.20 \pm 0.18$ & $\ldots$ \\
\hline B2259-375 & B2259-37 & $22-35$ & $7.47 \pm 0.16$ & $\ldots$ \\
\hline B2305-418 & B2305-41 & $\ldots$ & $4.02 \pm 0.18$ & $\ldots$ \\
\hline B2319-550 & B2319-55 & $\ldots$ & $5.73 \pm 0.25$ & $\ldots$ \\
\hline B2323-407 & B2323-40 & $23-43$ & $9.28 \pm 0.40$ & $\ldots$ \\
\hline B2331-416 & B2331-41 & $23-44$ & $15.92 \pm 0.69$ & $\ldots$ \\
\hline B2332-668 & B2332-66 & $23-63$ & $5.93 \pm 0.26$ & $\ldots$ \\
\hline B2338-585 & B2338-58 & $23-52$ & $8.17 \pm 0.21$ & $\ldots$ \\
\hline B2339-353 & B2339-353 & $\ldots$ & $5.67 \pm 0.22$ & 121 \\
\hline B2354-350 & B2354-35 & $23-37$ & $8.70 \pm 0.35$ & $\begin{array}{lllll}2 & 13 & 122 & 123 & 124\end{array}$ \\
\hline B2356-611 & & & & \\
\hline $\begin{array}{l}\text { B2356-612A } \\
\text { B2356-612B }\end{array}$ & B2356-61 & $23-64$ & $61.2 \pm 3.7$ & $\begin{array}{l}1681441114 \\
117125\end{array}$ \\
\hline
\end{tabular}


Table 4-Continued

\begin{tabular}{ccccc}
\hline \hline$(1)$ & $(2)$ & $(3)$ & $(4)$ & $(5)$ \\
MRC & PKS & MSH & $S_{408}$ & References for \\
name(s) & name(s) & name & $($ Jy $)$ & radio images \\
\hline
\end{tabular}

References. - (1) JM92; (2) Ekers et al. (1989); (3) Morganti et al. (1999b); (4) Slee (1977); (5) Duncan \& Sproats (1992); (6) SM75; (7) Danziger \& Goss (1983); (8) Subrahmanyan et al. (1996); (9) Saripalli et al. (2002); (10) Wall \& Schilizzi (1979); (11) Ekers et al. (1978); (12) Morganti et al. (1993); (13) Choi et al. (2004); (14) Christiansen et al. (1977); (15) White et al. (1984); (16) Jones (1989a); (17) Jones (1992); (18) Saripalli et al. (1994); (19) Sheridan (1958); (20) Shain (1958); (21) Heeschen (1961); (22) Gardner \& Whiteoak (1971); (23) Cameron (1971); (24) Ekers et al. (1983); (25) Geldzahler \& Fomalont (1984); (26) Fomalont et al. (1989); (27) Smith (1983); (28) Marvel et al. (1999); (29) Carter \& Malin (1983); (30) Harnett (1987); (31) Killeen \& Bicknell (1988); (32) Reid et al. (1999); (33) Reynolds et al. (1993); (34) Clarke et al. (1976); (35) McAdam et al. (1988); (36) Unewisse (1993); (37) Tingay et al. (1998); (38) Robertson \& Smith (1981); (39) Wall \& Cole (1973); (40) Swarup (1984); (41) Schwarz et al. (1974); (42) Slee \& Sheridan (1975); (43) Meisenheimer et al. (1989); (44) Perley et al. (1997); (45) Simkin et al. (1999); (46) Wardle et al. (1984); (47) Keel (1986); (48) Drinkwater et al. (1997); (49) Hardcastle et al. (1999); (50) Birkinshaw et al. (2002); (51) Parma et al. (1991); (52) Gregorini et al. (1994); (53) Otani et al. (1998); (54) Gliozzi et al. (1999); (55) Chartas et al. (2000); (56) Schwartz et al. (2000); (57) Rayner et al. (2000); (58) Ulvestad et al. (1981); (59) Safouris et al. (2003); (60) Sadler (1987); (61) O'Dea et al. (1994); (62) Taylor et al. (2002); (63) Bosma et al. (1983); (64) Harnett \& Reynolds (1985); (65) Whiteoak \& Bunton (1985); (66) Harnett et al. (1989); (67) Harnett et al. (1990); (68) Whiteoak \& Wilson (1990); (69) Dahlem et al. (1993); (70) Elmouttie et al. (1997); (71) Forbes \& Norris (1998); (72) Ott et al. (2001); (73) Smith \& Bicknell (1983); (74) Smith \& Bicknell (1986); (75) Lloyd et al. (1996); (76) Morganti et al. (2001); (77) McGee et al. (1955); (78) Bolton \& Clark (1960); (79) Cooper et al. (1965); (80) Lockhart \& Sheridan (1970); (81) Kaufmann et al. (1974); (82) Schreier et al. (1981); (83) Haslam et al. (1981); (84) Slee et al. (1983); (85) Burns et al. (1983); (86) Haynes et al. (1983); (87) Van Gorkom et al. (1990); (88) Clarke et al. (1992); (89) Tateyama \& Strauss (1992); (90) Junkes et al. (1993a); (91) Junkes et al. (1993b); (92) Schiminovich et al. (1994); (93) Condon et al. (1996); (94) Combi \& Romero (1997); (95) Morganti et al. (1999a); (96) Alvarez et al. (2000); (97) Kraft et al. (2002); (98) Sarma et al. (2002); (99) Hardcastle et al. (2003); (100) Kraft et al. (2003); (101) Killeen et al. (1986); (102) McAdam \& Schilizzi (1977); (103) Goss et al. (1987); (104) Subrahmanyan et al. (2003); (105) Saripalli et al. (2003); (106) Hunstead et al. (1982); (107) Sault \& Wieringa (1994); (108) de Pater et al. (1985); (109) Villar-Martín et al. (1998); (110) De Breuck et al. (2000); (111) Goss et al. (1982); (112) Röttgering et al. (1997); (113) Lloyd \& Jones (2002); (114) Schwarz et al. (1973); (115) Tadhunter et al. (1988); (116) Norris et al. (1990); (117) Ekers \& Whiteoak (1992); (118) Fosbury et al. (1998); (119) Fosbury et al. (1982); (120) Clark et al. (1997); (121) De Breuck et al. (2004); (122) Schwartz et al. (1991); (123) Taylor et al. (1994); (124) Heinz et al. (2002); (125) Sadler (1997). 
Table 5. Radio positions, flux densities, angular sizes, and position angles for the MS4 sample.

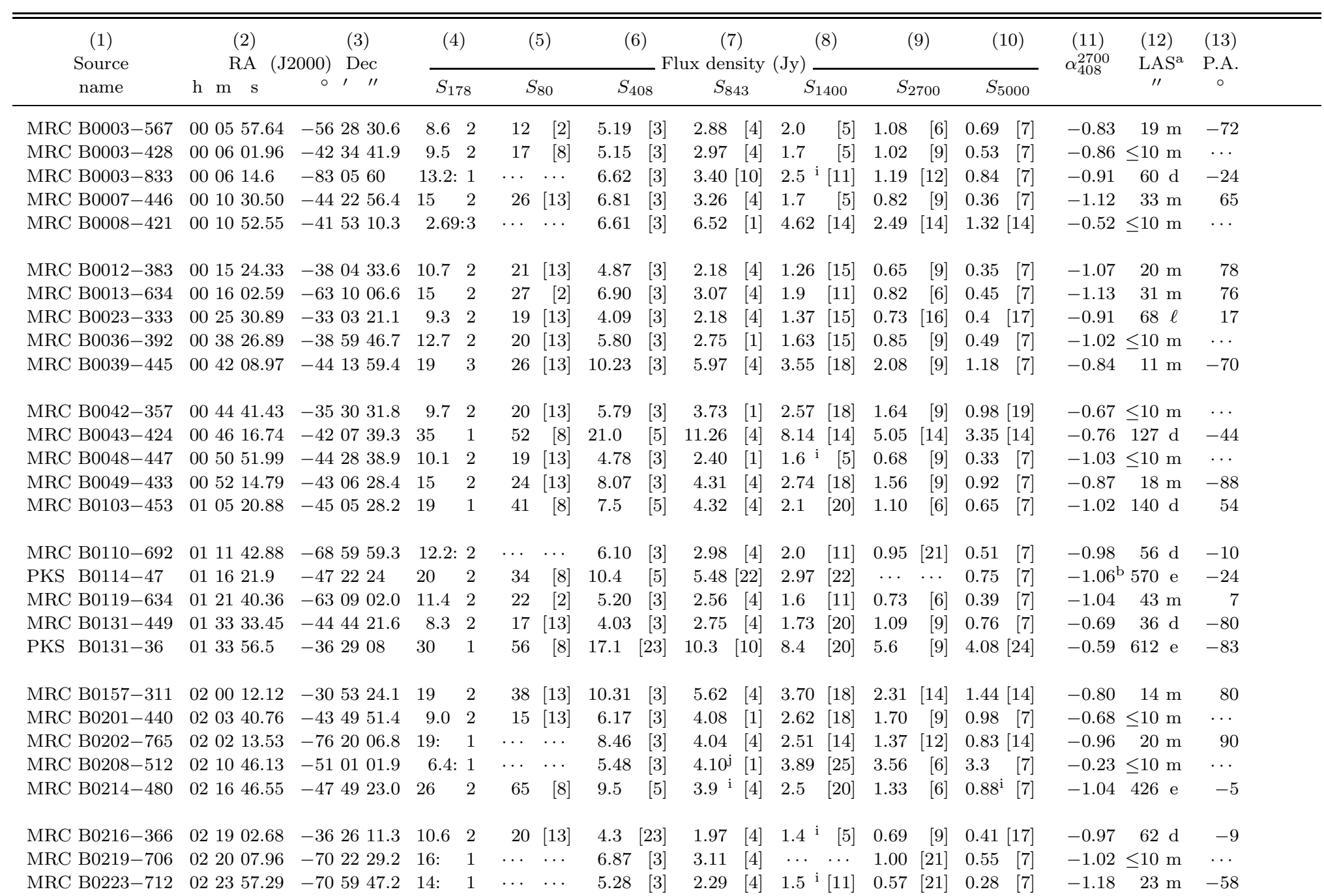


Table 5-Continued

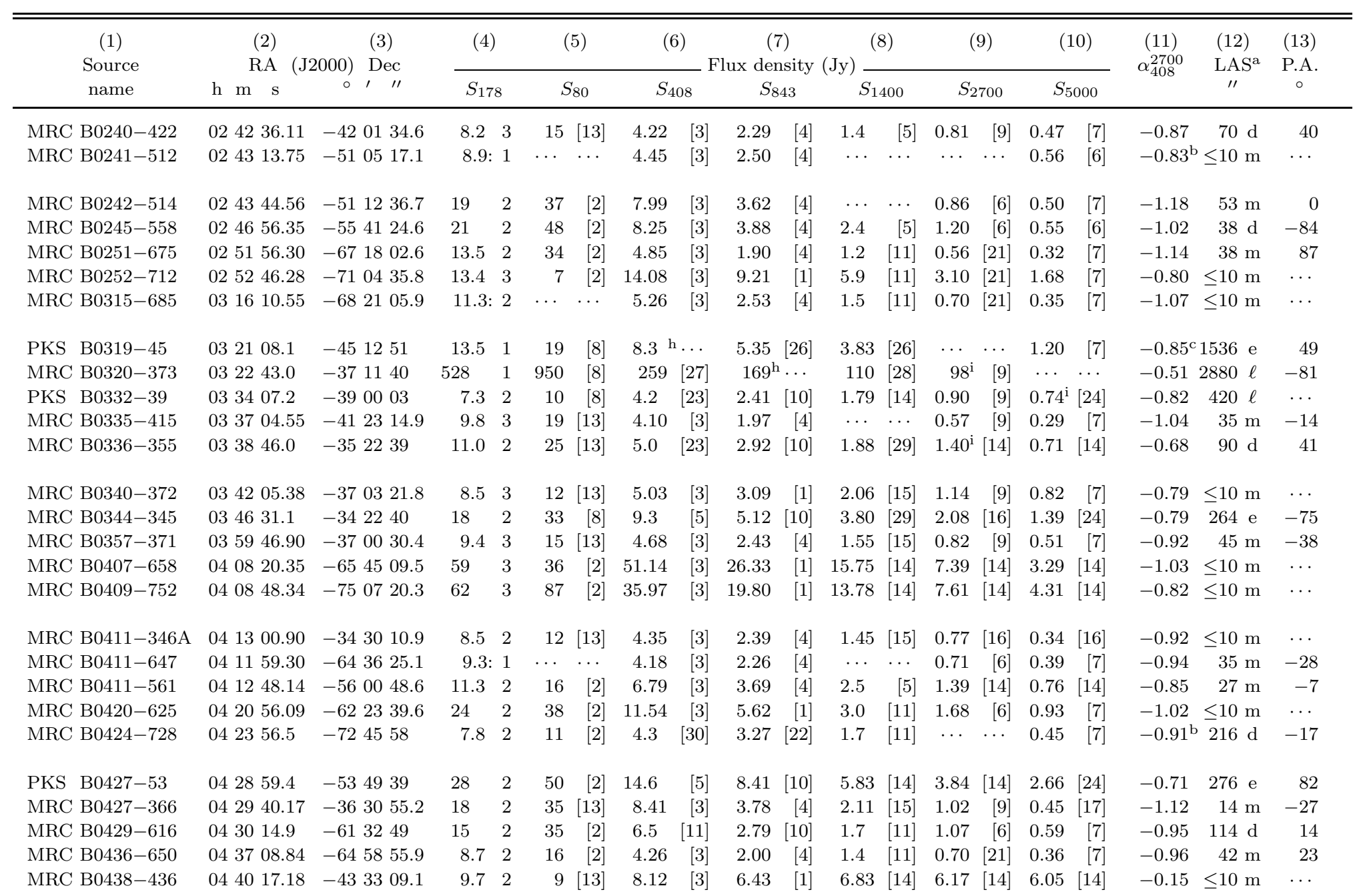


Table 5-Continued

\begin{tabular}{|c|c|c|c|c|c|c|c|c|c|c|c|c|c|c|}
\hline \multirow{3}{*}{$\begin{array}{c}\begin{array}{c}(1) \\
\text { Source } \\
\text { name }\end{array} \\
\text { MRC B0453-301 }\end{array}$} & $(2)$ & \multirow{2}{*}{\multicolumn{2}{|c|}{$\begin{aligned} & (3) \\
000) & \text { Dec } \\
\circ, & \prime \prime\end{aligned}$}} & \multicolumn{2}{|c|}{ (4) } & $(5)$ & (6) & $(7)$ & $(8)$ & (9) & (10) & \multirow{2}{*}{$\begin{array}{c}(11) \\
\alpha_{408}^{2700}\end{array}$} & \multirow{2}{*}{$\begin{array}{l}(12) \\
\mathrm{LAS}^{\mathrm{a}} \\
\prime \prime\end{array}$} & \multirow{2}{*}{$\begin{array}{l}\text { (13) } \\
\text { P.A. } \\
\circ\end{array}$} \\
\hline & $\mathrm{h} \mathrm{m} \mathrm{s}$ & & & $S_{178}$ & & $S_{80}$ & $S_{408}$ & $S_{843}$ & $S_{1400}$ & $S_{2700}$ & $S_{5000}$ & & & \\
\hline & 045514.33 & -30 & 0648.5 & 18 & 2 & $31[13]$ & $9.16 \quad[3]$ & $5.07 \quad[4]$ & $3.31[18]$ & $1.93[16]$ & $1.19[19]$ & -0.82 & $50 \mathrm{~m}$ & -1 \\
\hline MRC B0454-463 & 045550.76 & -46 & 1559.0 & 9.3 & 2 & $13[13]$ & $4.25[3]$ & $3.00[4]$ & $2.22[18]$ & $2.36^{\mathrm{i}}[6]$ & $1.57^{\mathrm{i}}[7]$ & -0.31 & $\leq 10 \mathrm{~m}$ & $\ldots$ \\
\hline MRC B0456-301 & 045826.43 & -30 & 0722.4 & 13.3 & 2 & $27[13]$ & $7.2 \quad[31]$ & $3.89 \quad[4]$ & $2.6 \quad[20]$ & $1.67[16]$ & $0.90^{\mathrm{i}}[19]$ & -0.77 & $348 \ell$ & 2 \\
\hline MRC B0506-612 & 050643.80 & -61 & 0941.0 & 8.8 & 2 & $14 \quad[2]$ & $5.03[3]$ & $2.96[4]$ & $2.2 \quad[11]$ & $1.5 \quad[5]$ & $1.17 \quad[7]$ & -0.65 & $\leq 10 \mathrm{~m}$ & $\cdots$ \\
\hline MRC B0509-573 & 051018.55 & -57 & 1941.5 & 10.3: & 1 & $\ldots \quad \ldots$ & 5.12 & $2.63[1]$ & $1.64[25]$ & $0.97 \quad[6]$ & $0.60 \quad[7]$ & -0.88 & $\overline{\leq} 10 \mathrm{~m}$ & $\cdots$ \\
\hline MRC B0511-484 & $05 \quad 1250.79$ & -48 & 2404.3 & 12.2: & 1 & $\ldots$ & $6.84 \quad[3]$ & $4.44 \quad[4]$ & $3.3 \quad[20]$ & $1.80 \quad[6]$ & $1.81^{\mathrm{i}}[24]$ & -0.71 & $132 \mathrm{~d}$ & 70 \\
\hline PKS B0511-30 & 051334.6 & -30 & 2824 & 16 & 2 & $29 \quad[8]$ & $7.8 \quad[23]$ & $4.90[22]$ & $3.65[14]$ & $3.21^{\mathrm{i}}[16]$ & $1.48[14]$ & -0.47 & $636 \mathrm{e}$ & 27 \\
\hline MRC B0513-488 & $05 \quad 1433.20$ & -48 & 4530.5 & 9.3: & 1 & $\ldots \quad \ldots$ & $4.04 \quad[3]$ & $1.87 \quad[4]$ & $\ldots \quad \ldots$ & $0.64 \quad[6]$ & $0.32 \quad[7]$ & -0.98 & $43 \mathrm{~m}$ & -38 \\
\hline PKS B0518-45 & 051946.0 & -45 & 4633 & 349 & 2 & $570 \quad[8]$ & 166 & $85.7 \mathrm{i}[10]$ & 68.8 & $29 \quad[6]$ & 15 & -0.92 & $432 \mathrm{e}$ & -77 \\
\hline MRC B0521-365 & 052257.97 & -36 & 2728.2 & 61 & 1 & $99[13]$ & $36.08 \quad[3]$ & $22.80 \quad[4]$ & $17.50[14]$ & $11.71[14]$ & $8.49[14]$ & -0.60 & $19 \mathrm{~m}$ & -72 \\
\hline MRC B0534-497 & 053613.69 & -49 & 4421.6 & 11.9 & 2 & $16 \quad[8]$ & 6.69 & $3.29 \quad[4]$ & 2.1 & 1.0 & $0.58 \quad[7]$ & -1.02 & $30 \mathrm{~m}$ & 60 \\
\hline MRC B0546-445 & 054737.98 & -44 & 3112.0 & 11.4 & 2 & 31 [13] & $4.41[3]$ & $2.56[4]$ & 1.5 & $0.71[9]$ & $0.41[7]$ & -0.97 & $14 \mathrm{~m}$ & 3 \\
\hline MRC B0547-408 & 054923.57 & -40 & 5114.6 & 16 & 2 & $28[13]$ & 8.24 & $4.72 \quad[4]$ & $2.55[18]$ & $1.29[9]$ & $0.87 \quad[7]$ & -0.98 & $37 \mathrm{~m}$ & -63 \\
\hline MRC B0601-344 & 060311.85 & -34 & 2640.7 & 10.4 & 2 & $20[13]$ & $4.40 \quad[3]$ & 2.52 & $1.7 \quad[5]$ & $0.83[16]$ & $0.41^{\mathrm{i}}[16]$ & -0.88 & $64 \mathrm{~d}$ & 30 \\
\hline MRC B0602-319 & 060414.53 & -31 & 5557.6 & 10.8 & 2 & $19[13]$ & $6.67 \quad[3]$ & $3.93 \quad[4]$ & $2.93[18]$ & $1.89[16]$ & $1.18^{\mathrm{i}}[19]$ & -0.67 & $12 \mathrm{~m}$ & $\ldots$ \\
\hline MRC B0602-647 & 060238.65 & -64 & 4329.4 & 7.1: & 2 & $\ldots \quad \ldots$ & $4.49 \quad[3]$ & $2.65[4]$ & $1.4[11]$ & $0.70[32]$ & $0.31 \quad[7]$ & -0.98 & $70 \mathrm{~m}$ & 8 \\
\hline MRC B0614-349 & $06 \quad 1635.94$ & -34 & 5617.6 & 7.6 & 2 & $11[13]$ & $5.29 \quad[3]$ & $3.73[1]$ & $2.91[14]$ & $1.91[14]$ & $1.35[14]$ & -0.54 & $\leq 10 \mathrm{~m}$ & $\ldots$ \\
\hline MRC B0615-365 & $06 \quad 1732.19$ & -36 & 3415.2 & 6.8 & 2 & $11[13]$ & $4.41[3]$ & $2.92 \quad[4]$ & $2.07[15]$ & $1.17 \quad[9]$ & $0.65[9]$ & -0.70 & $\leq 10 \mathrm{~m}$ & $\ldots$ \\
\hline MRC B0618-371 & 062000.55 & -37 & 1140.8 & 10.7 & 2 & $18[13]$ & $5.81[33]$ & $3.80 \quad[4]$ & $2.97 \quad[14]$ & $1.94[14]$ & $1.31[14]$ & -0.59 & $78 \mathrm{~d}$ & 81 \\
\hline MRC B0620-526 & 062141.53 & -52 & 4115.5 & 17 & 2 & $30 \quad[2]$ & $9.3 \quad[5]$ & $5.56 \quad[4]$ & $3.4 \quad[5]$ & $2.1 \quad[5]$ & $1.37 \quad[7]$ & -0.80 & $318 \ell$ & $\cdots$ \\
\hline MRC B0625-536 & 062622.14 & -53 & 4127.1 & 56 & 2 & $113[2]$ & [5] & $11.60 \quad[4]$ & 6.7 & $3.70[32]$ & $2.1 \quad[7]$ & -1.03 & $118 \ell$ & 1 \\
\hline MRC B0625-354 & 062706.83 & -35 & 2914.0 & 18 & 2 & $33[13]$ & $9.2 \quad[31]$ & $5.86 \quad[4]$ & $4.88[14]$ & $2.90[9]$ & $2.27^{\mathrm{i}}[14]$ & -0.61 & $118 \ell$ & $\cdots$ \\
\hline MRC B0625-545 & 062647.21 & -54 & 3238.9 & 12.1: & 2 & $\ldots \quad \ldots$ & $7.9[31]$ & $5.00 \quad[4]$ & $3.2[34]$ & $1.73[32]$ & $0.93[7]$ & -0.80 & $330 \ell$ & -4 \\
\hline MRC B0637-752 & $06 \quad 3545.73$ & -75 & 1615.8 & 12.5 & 2 & $20 \quad[2]$ & $7.89 \quad[3]$ & $6.67^{\mathrm{i}}[4]$ & $5.33[14]$ & $5.16[14]$ & $5.36[14]$ & -0.22 & $13 \mathrm{~m}$ & 90 \\
\hline MRC B0646-398 & 064811.30 & -39 & 5707.3 & 13.9 & 2 & $26[13]$ & $7.0 \quad[5]$ & 3.85 [4] & $2.63[18]$ & $1.53 \quad[9]$ & $1.03[7]$ & -0.80 & $82 \mathrm{~d}$ & -5 \\
\hline MRC B0647-475 & 064848.36 & -47 & 3428.0 & 5.8: & 2 & $\ldots \quad \ldots$ & $4.35 \quad[3]$ & $3.22 \quad[4]$ & $\ldots$ & $1.14[32]$ & $0.68 \quad[7]$ & -0.71 & $\leq 10 \mathrm{~m}$ & $\ldots$ \\
\hline MRC B0658-656 & $0658 \quad 12.84$ & -65 & 4453.3 & 11.0 & 2 & $17 \quad[2]$ & $6.14 \quad[3]$ & $3.20 \quad[4]$ & {$[11]$} & $1.10[21]$ & $0.59 \quad[7]$ & -0.91 & $25 \mathrm{~m}$ & 90 \\
\hline MRC B0700-473 & $0702 \quad 09.13$ & -47 & 2633.5 & 9.2 & 2 & $14[13]$ & 4.33 & $1.85[4]$ & 1.1 & $0.60[32]$ & 0.27 & -1.05 & $40 \mathrm{~m}$ & -56 \\
\hline
\end{tabular}


Table 5-Continued

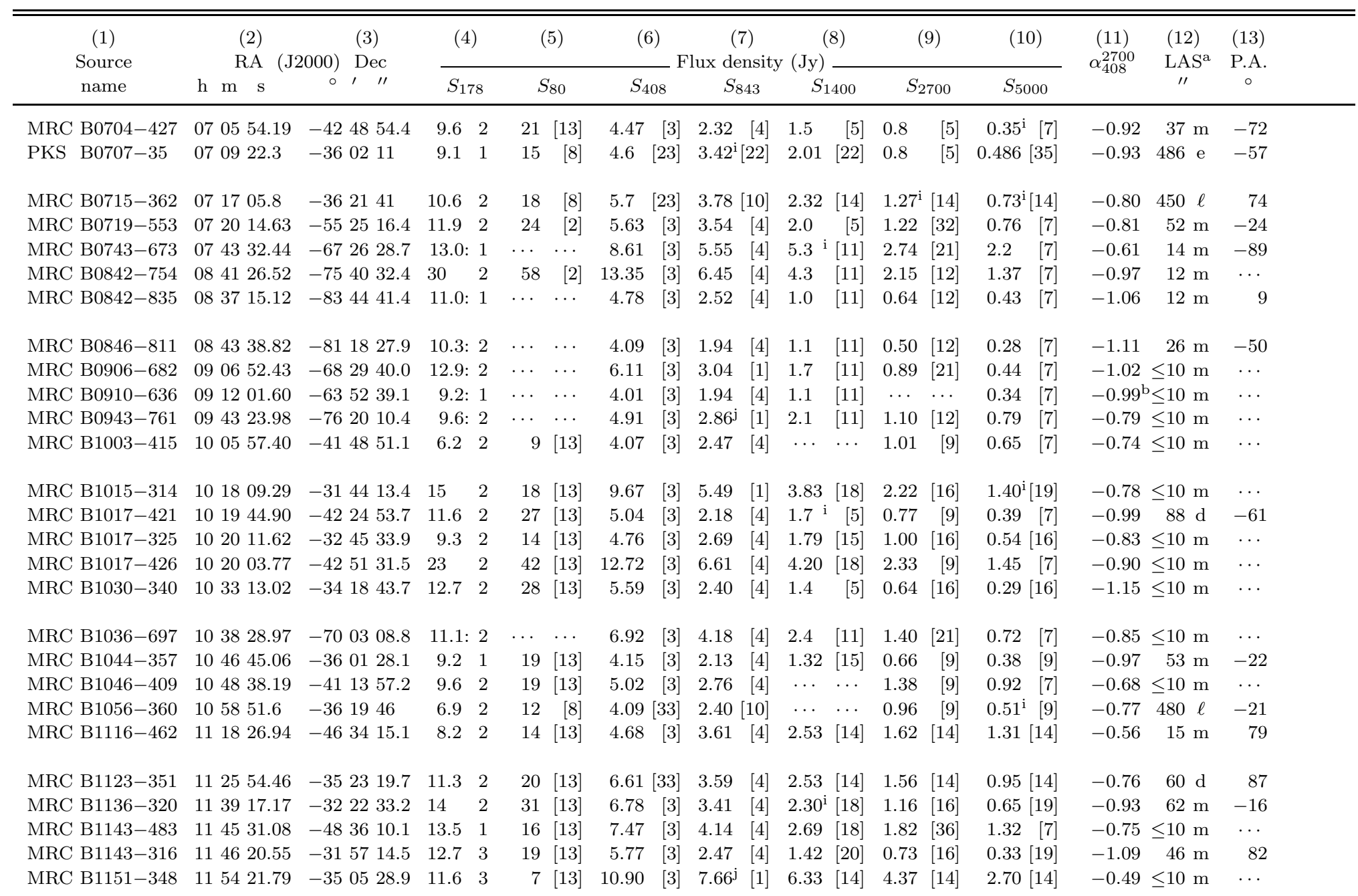


Table 5-Continued

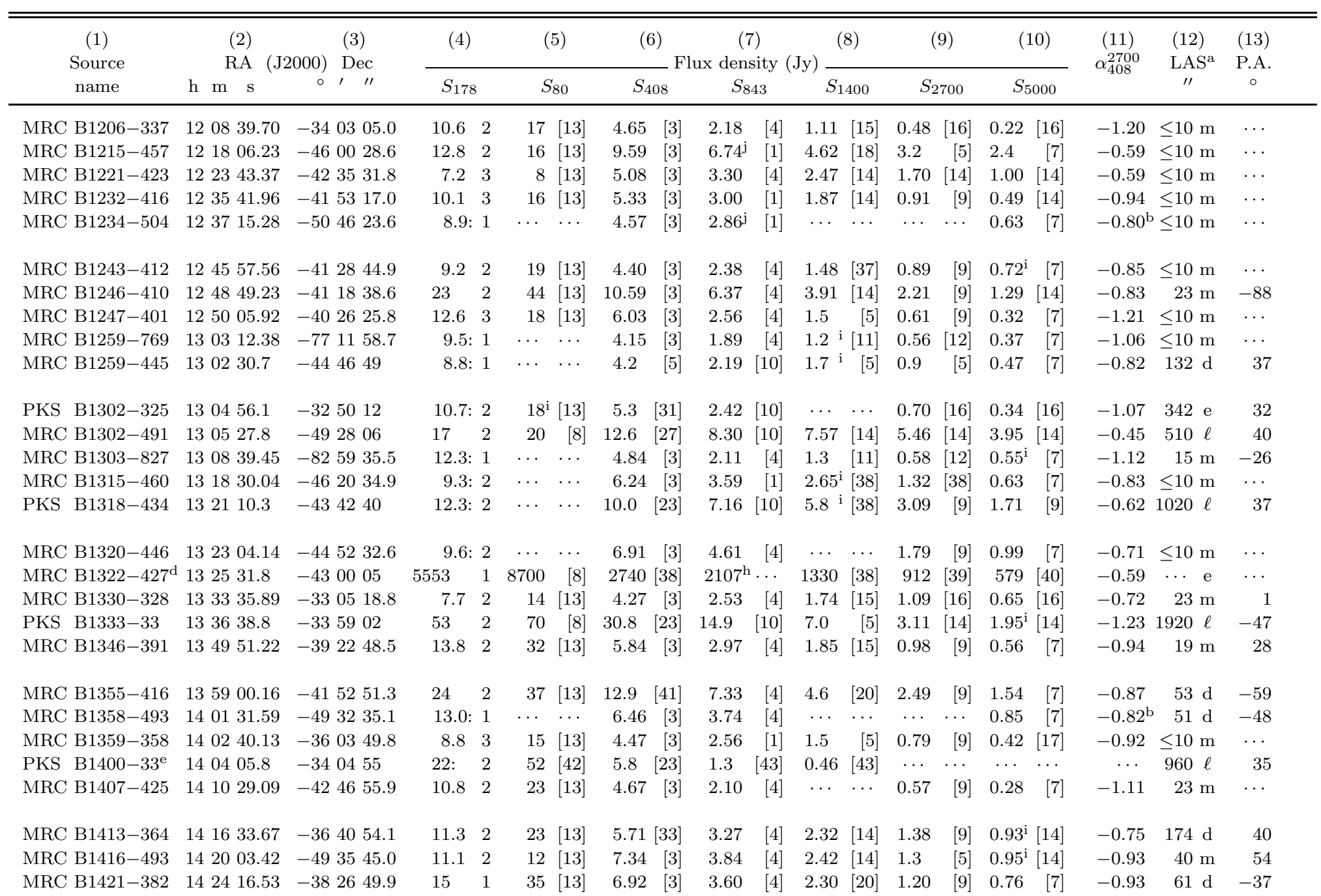


Table 5-Continued

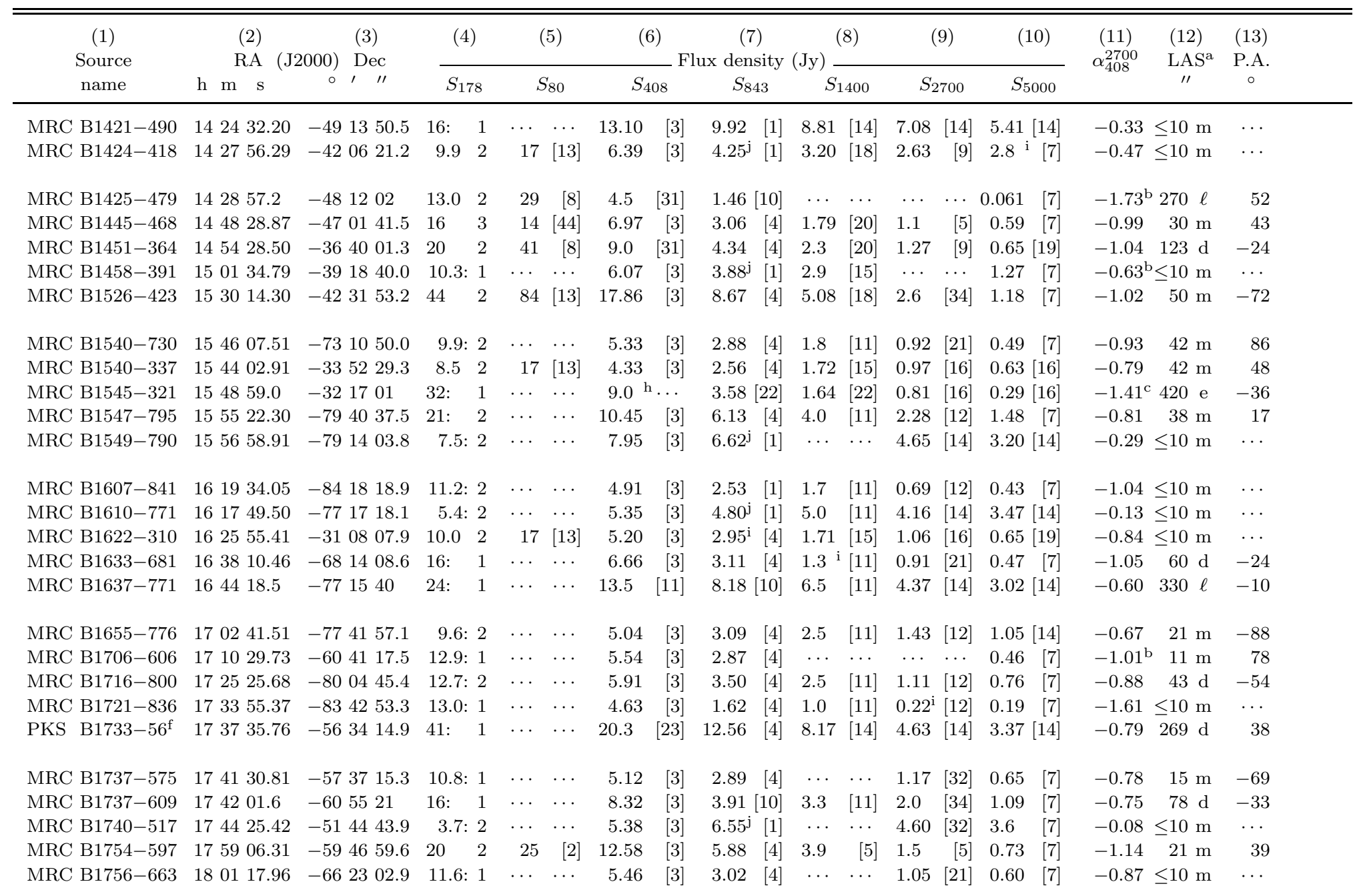


Table 5-Continued

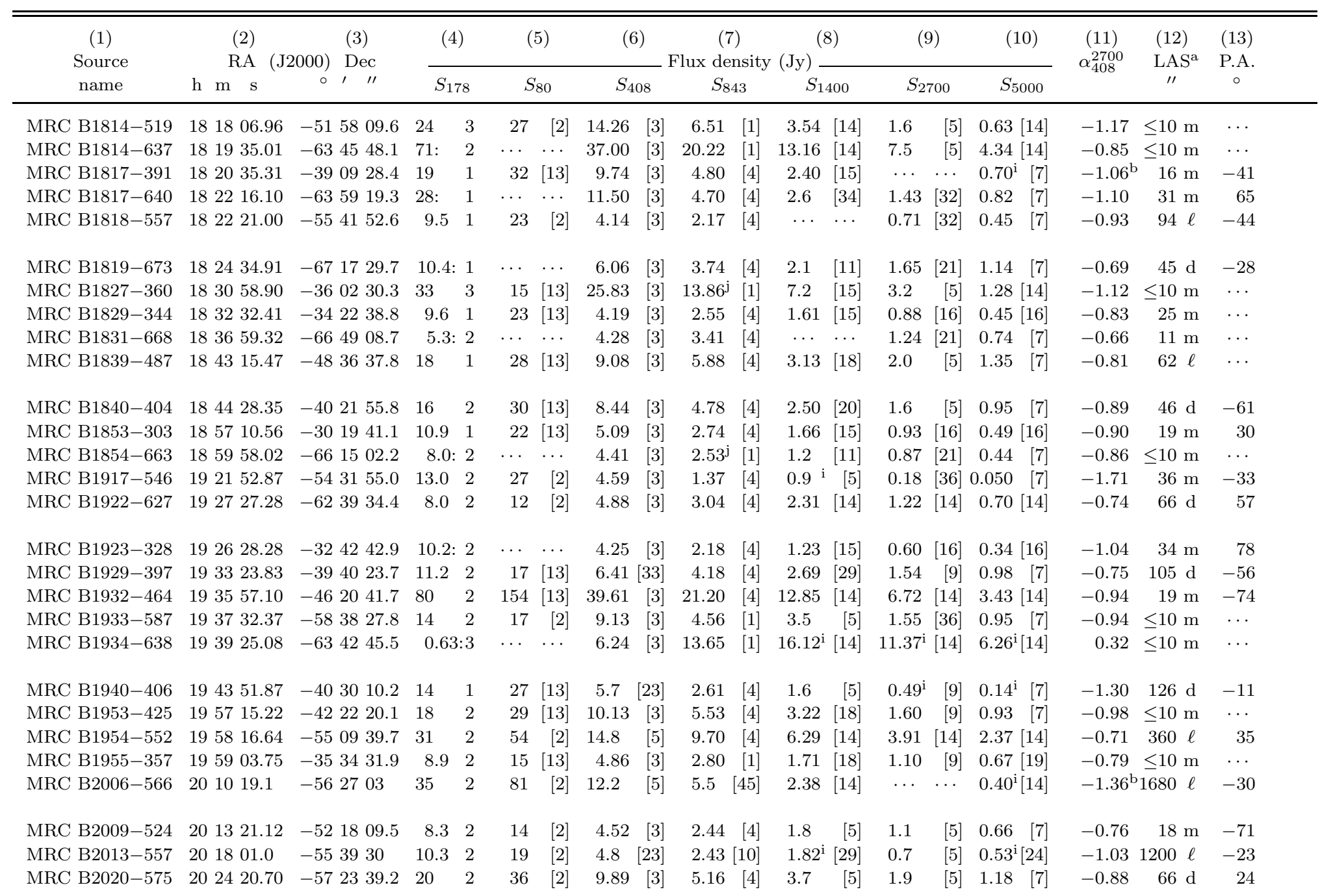


Table 5-Continued

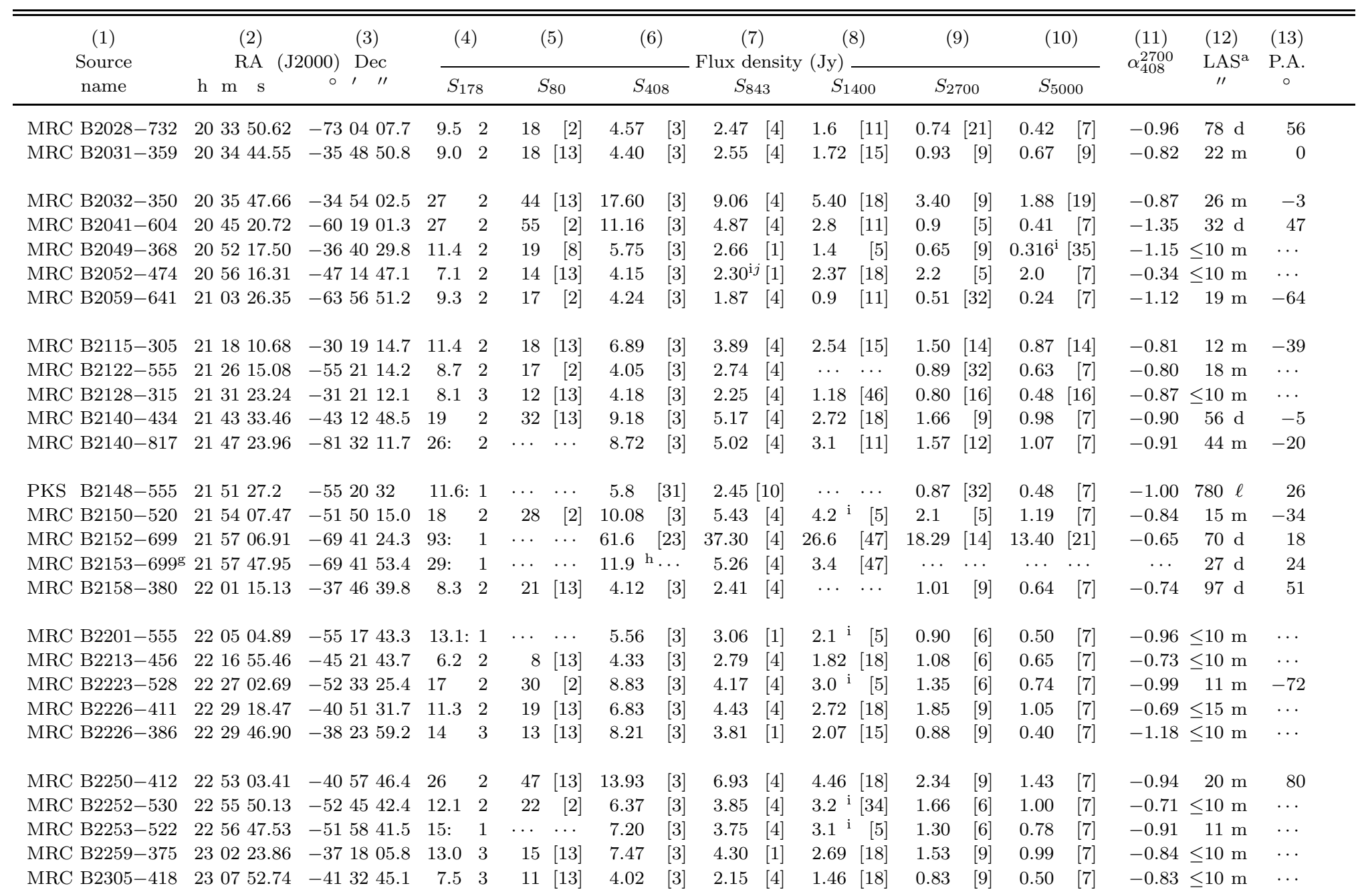


Table 5-Continued

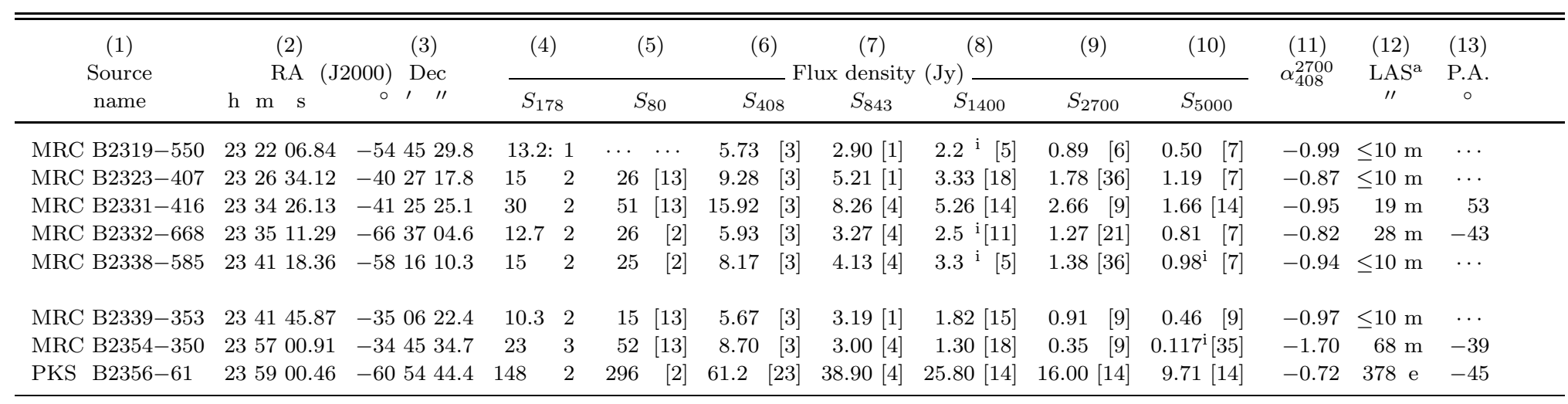

aSize flags: (d) separation of peaks of a double source; (e) largest separation of peaks of a complex source; $(\ell)$ largest size of low-surface-brightness emission in a complex source: the position angle given is that of the central axis; (m) deconvolved extent along the axis of a source, as in JM92.

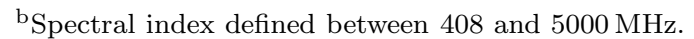

${ }^{\mathrm{c} S p e c t r a l}$ index defined between 843 and $5000 \mathrm{MHz}$.

${ }^{\mathrm{d}}$ The total angular extent is almost $10^{\circ}$ (e.g. Cooper et al. 1965), whereas that of the inner lobes is $6^{\prime} .8$ (JM92). The position angle of the structure varies at different angular scales: for the inner lobes it is $49^{\circ}$ (JM92).

eThe position, largest angular extent and position angle are the $408 \mathrm{MHz}$ values from McAdam \& Schilizzi (1977).

${ }^{\mathrm{f}}$ The position is that of the core in the MOST image.

g Angular size and position angle were measured from an ATCA contour map (Norris et al. 1990), as the MOST CUTS were affected by MRC B2152-699.

${ }^{\mathrm{h}}$ Flux density estimated from the radio spectrum.

${ }^{\mathrm{i}}$ Flux density not used in estimating $S_{178 \mathrm{MHz}}$.

jVariable at $843 \mathrm{MHz}$ (Gaensler \& Hunstead 2000).

References. - (1) Campbell-Wilson \& Hunstead (1994); (2) Mills et al. (1961); (3) Large et al. (1981); (4) this paper; (5) Bolton et al. (1964); (6) Wright et al. (1977); (7) Gregory et al. (1994); (8) Mills et al. (1960); (9) Bolton \& Shimmins (1973); (10) JM92; (11) Price \& Milne (1965); (12) Shimmins \& Bolton (1972b); (13) Slee (1995); (14) Wills (1975); (15) Condon et al. (1998); (16) Shimmins \& Bolton (1974); (17) Shimmins \& Bolton (1972a); (18) Fomalont \& Moffet (1971); (19) Shimmins et al. (1969); (20) Fomalont (1968); (21) Bolton \& Butler (1975); (22) Subrahmanyan et al. (1996); (23) SM75; (24) Wall \& Schilizzi (1979); (25) Quiniento et al. (1988); (26) Saripalli et al. (1994); (27) Cameron (1971); (28) Goldstein (1962); (29) Smith (1983); (30) Clarke et al.

Shilizi (1979); (25) Quiniento et al. (1988); (26) Saripali et al. (1994); (27) Cameron (1971); (28) Goldstein (1962); (29) Smith (1983); (30) Clarke et al. 
(1976); (31) Molonglo Transit Catalogue; (32) Wall et al. (1975); (33) Ekers et al. (1989); (34) Gardner et al. (1969); (35) Wright et al. (1996); (36) Shimmins (1971); (37) Quiniento \& Echave (1990); (38) Cooper et al. (1965); (39) Rogstad \& Ekers (1969); (40) Junkes et al. (1993a), reduced by 15\% (Alvarez et al. 2000); (41) Wyllie (1969b); (42) McAdam \& Schilizzi (1977); (43) Subrahmanyan et al. (2003); (44) Slee \& Higgins (1973); (45) Röttgering et al. (1997);

(46) Quiniento \& Cersosimo (1993); (47) Christiansen et al. (1977). 
Table 6. Sample size, source density, and median values of various quantities for sources in the MS4, SMS4, and 3CRR samples.

\begin{tabular}{rrrrr}
\hline \hline (1) & $(2)$ & $\begin{array}{c}(3) \\
\text { Source } \\
\text { Sample }\end{array}$ & $\mathrm{N}$ & \multicolumn{2}{c}{$\begin{array}{c}\text { Median values } \\
\text { density } \\
\left(\mathrm{sr}^{-1}\right)\end{array}$} & $\begin{array}{c}S_{178} \\
(\mathrm{Jy})\end{array}$ & $\alpha$ \\
\hline & & & & \\
MS4 & 228 & $94.0 \pm 6.2$ & $12.1_{-0.7}^{+0.6}$ & $-0.86_{-0.02}^{+0.03}$ \\
SMS4 & 137 & $56.5 \pm 4.8$ & $15.7_{-0.7}^{+0.5}$ & $-0.91_{-0.02}^{+0.04}$ \\
3CRR & 172 & $40.6 \pm 3.1$ & $15.6_{-0.8}^{+0.8}$ & $-0.81_{-0.01}^{+0.03}$ \\
\hline
\end{tabular}

